\title{
Covering games and the Banach-Mazur game: $k$-tactics.
}

\author{
Tomek Bartoszynski* \\ Department of Mathematics, \\ Boise State University, \\ Boise, Idaho 83725
}

\author{
Winfried Just ${ }^{\dagger}$ \\ Department of Mathematics, \\ Ohio University, \\ Athens, Ohio 45701
}

\author{
Marion Scheepers ${ }^{\ddagger}$ \\ Department of Mathematics, \\ Boise State University, \\ Boise, Idaho 83725
}

\begin{abstract}
Given a free ideal $J$ of subsets of a set $X$, we consider games where player ONE plays an increasing sequence of elements of the $\sigma$-completion of $J$, and player TWO tries to cover the union of this sequence by playing one set at a time from $J$. We describe various conditions under which player TWO has a winning strategy that uses only information about the most recent $k$ moves of ONE, and apply some of these results to the Banach-Mazur game.
\end{abstract}

\section{Introduction}

Let $J$ be a free ideal of subsets of a given set. By $\langle J\rangle$ we denote the $\sigma$ ideal generated by $J(\langle J\rangle$ could turn out to be the power set of $\cup J)$. Two concrete examples of ideals motivated much of our work. The one is $J_{\mathbb{R}}$, the ideal of nowhere dense subsets of the real line $\mathbb{R}$. In this case $\left\langle J_{\mathbb{R}}\right\rangle$ is the ideal of meager sets of reals. The other is $[\kappa]^{<\lambda}$ where $\omega=\operatorname{cof}(\lambda) \leq \lambda \leq \kappa$ are cardinal numbers.

We are interested in games of the following type: Player ONE plays a set $O_{n} \in\langle J\rangle$ during inning $n$, to which TWO responds with a set

\footnotetext{
* Supported by Idaho State Board of Education grant 92-096

† Supported by NSF grant DMS-9016021 and Research Challenge Grant RC 89-64 from Ohio University

${ }^{\ddagger}$ Supported by Idaho State Board of Education grant 91-093.
} 
$T_{n} \in J$. ONE is required to play an increasing sequence of sets; TWO's objective is to cover $\bigcup_{n \in \omega} O_{n}$ with $\bigcup_{n \in \omega} T_{n}$. As long as TWO remembers the complete history of the game, this task is trivial. However, it often happens that TWO needs to know only the last $k$ moves of the opponent in order to win. A strategy that accomplishes this is called a winning $k$-tactic.

We consider four such games, $M G(\mathcal{A}, J), M G(J)$, the "monotonic game", $S M G(J)$, the "strongly monotonic game", and $V S G(J)$, the "very strong game". The study of these games was initiated in [S1], and motivated by Telgarský's conjecture that for every $k>0$ there exists a topological space $(X, \tau)$ such that TWO has a winning $k+1$-tactic but no winning $k$-tactic in the Banach-Mazur game on $(X, \tau)$ (see section 4.4 for more information). However, we find the games considered here of interest independent of the original motivation. The game $M G(J)$ was introduced in $\mathrm{S} 1]$, as was the game $S M G(J)$; the games $M G(\mathcal{A}, J)$ and $V S G(J)$ appear here for the first time.

In sections 2 and 3, we introduce and discuss pseudo Lusin sets, the irredundancy property and the coherent decomposition property of ideals. These properties, together with the $\omega$-path partition relation, are the main tools for constructing winning $k$-tactics in our games. These combinatorial properties of ideals are very likely of independent interest - they have already appeared in the literature in various guises.

In section 4 we apply the results of sections 2 and 3 to give various conditions sufficient for the existence of winning $k$-tactics for TWO in the games mentioned above. Not surprisingly, as the game becomes more favorable for TWO, weaker conditions suffice. Among other things, our results show that in the Banach-Mazur game on the space that inspired the invention of meager-nowhere dense games, TWO has a winning 2-tactic.

The appendix is devoted to a proof of an unpublished consistency result of Stevo Todorcevic, which we use in section 4 .

Our notation is mostly standard. One important exception may be that we use the symbol $\subset$ exclusively to mean "is a proper subset of". Where we otherwise deviate from standard notation or terminology we explicitly alert the reader. For convenience we also assume the consistency of traditional (Zermelo-Fraenkel) set theory. All statements we make about the consistency of various mathematical assertions must be understood as consistency which can be proven by means of that theory. The reader might find having a copy of [S1] and [S2] handy when reading parts of this paper a bit more comfortable than otherwise.

We are grateful to Stevo Todorčević for sharing with us his insights about the matters we study here, and for his kind permission to present in this paper some of his answers to our questions. 


\section{The irredundancy property.}

For a partially ordered set $(P,<)$ which has no maximum element we let

$$
\operatorname{add}(P,<)
$$

be the least cardinal number, $\lambda$, for which there is a collection of cardinality $\lambda$ of elements of $P$ which do not have an upper bound in $P$. This cardinal number is said to be the additivity of $(P,<)$. Note that add $(P,<)$ is either 2 , or else it is infinite. In the latter case $(P,<)$ is said to be $d i$ rected. We attend exclusively to directed partially ordered sets in this paper. Isbell [n] and some earlier authors also refer to the additivity of a partially ordered set as its lower character; they denote it by $\ell(P,<)$.

A free ideal $J$ on a set $S$ is partially ordered by $\subset$. The partially ordered set $(J, \subset)$ is directed. When $\operatorname{add}(J, \subset)=\aleph_{0}$, the symbol $\langle J\rangle$ denotes the $\sigma$-completion of $J$ (i.e., the smallest collection which contains each union of countably many sets from $J$ ). We say that $J$ is a $\sigma$-complete ideal if $J=\langle J\rangle$.

The other important example for our study is the set ${ }^{\omega} \omega$ of sequences of nonnegative integers; we use $\mathfrak{c}$ to denote the cardinality of this set. We say $g$ eventually dominates $f$ and write $f \ll g$ if: $\lim _{n \rightarrow \infty}(g(n)-f(n))=\infty$. It is customary to denote $\operatorname{add}\left({ }^{\omega} \omega, \ll\right)$ by $\mathfrak{b}$.

A well known theorem of Miller ( $\mathrm{M}$, p. 94, Theorem 1.2) states that

$$
\operatorname{add}\left(\left\langle J_{\mathbb{R}}\right\rangle, \subset\right) \leq \operatorname{add}\left({ }^{\omega} \omega, \ll\right)(=\mathfrak{b}) .
$$

Again, for an arbitrary partially ordered set $(P,<)$ the symbol

$$
\operatorname{cof}(P,<)
$$

denotes the least cardinal number, $\kappa$, for which there is a collection $X$ of cardinality $\kappa$ of elements of $P$ such that: for each $p \in P$ there is an $x \in X$ such that $p \leq x$. This cardinal number is said to be the cofinality of $(P,<)$. Some authors (see e.g. [I], p. 397) also call this cardinal number the upper character of $(P,<)$ and denote it by $u(P,<)$. It is customary to denote $\operatorname{cof}\left({ }^{\omega} \omega, \ll\right)$ by $\mathfrak{d}$.

A theorem of Fremlin ([F], Proposition 13(b)) states that

$$
(\mathfrak{d}=) \operatorname{cof}\left({ }^{\omega} \omega, \ll\right) \leq \operatorname{cof}\left(\left\langle J_{\mathbb{R}}\right\rangle, \subset\right) .
$$

Let $(P,<)$ be a directed partially ordered set. The bursting number of $(P,<)([\mathbb{I}]$, p. 401) is the smallest cardinal number which exceeds the cardinality of each of the bounded subsets of $(P,<)$. This cardinal number is denoted by $\operatorname{burst}(P,<)$. More important is the principal bursting number of $(P,<)$, denoted $b u(P,<)$ and define as

$$
b u(P,<)=\min \{\operatorname{burst}(Q,<): Q \text { is a cofinal subset of } P\}
$$

(following [I], p. 409). It is always the case that $\operatorname{add}(P,<) \leq b u(P,<)$. 
Definition $1 A$ directed partially ordered set $(P,<)$ has the irredundancy property if:

$$
b u(P,<)=\operatorname{add}(P,<) .
$$

The cofinal subfamily $\mathcal{A}$ of $(P,<)$ is said to be irredundant if burst $(\mathcal{A},<$ )$\leq \operatorname{add}(P,<)$.

Not all $\sigma$-complete ideals have the irredundancy property. Here is an ad hoc example. Let $S_{1}$ and $S_{2}$ be disjoint sets such that $S_{i}$ has cardinality $\aleph_{i}$ for each $i$. Define an ideal $J$ on the union of these sets by admitting a set $Y$ into $J$ if: $Y \cap S_{1}$ is countable and $Y \cap S_{2}$ has cardinality less than $\aleph_{2}$. Then $\operatorname{add}(J, \subset)=\aleph_{1}$ and $\operatorname{cof}(J, \subset)=\aleph_{2}$. No cofinal family of $J$ is irredundant.

A refined version of the classical notion of a Lusin set is instrumental in verifying the presence of the irredundancy property in many directed partially ordered sets. Since what we'll define is not exactly the same as the classical notion, we call our "Lusin sets" pseudo Lusin sets (more about this after the definition). Let $\kappa$ and $\lambda$ be infinite cardinal numbers. Let $(P,<)$ be a directed partially ordered set.

Definition $2 A$ subset $L$ of $P$ is a $(\kappa, \lambda)$ pseudo Lusin set if:

1. $\lambda$ is the cardinality of $L$ and

2. for each $x \in P$ the cardinality of the set $\{y \in L: y \leq x\}$ is less than $\kappa$.

$(\kappa, \lambda)$ pseudo Lusin sets are interesting only when $\kappa \leq \lambda$. If a directed partially ordered set $(P,<)$ has a $(\kappa, \lambda)$ pseudo Lusin set, then $\operatorname{add}(P,<$ )$\leq \kappa$ and $\lambda \leq \operatorname{cof}(P,<)$. Moreover, every partially ordered set has an $(\operatorname{add}(P,<), \operatorname{add}(P,<))$ pseudo Lusin set. Thus, if $\operatorname{add}(P,<)=\operatorname{cof}(P,<)$, then these are the only types of pseudo Lusin sets in $(P,<)$.

Let $J$ be a free ideal on a set $S$. The uniformity number of $J$, written unif $(J)$, is the minimal cardinal $\kappa$ such that there is a subset of $S$ which is of cardinality $\kappa$, which is not an element of $J$.

Consider the partially ordered set $\left(\left\langle J_{\mathbb{R}}\right\rangle, \subset\right)$. If $L \subset \mathbb{R}$ is a Lusin set in the classical sense (i.e., $L$ is uncountable and every meager set meets $L$ in only countably many points), then $\{\{x\}: x \in L\}$ is an $\left(\omega_{1},|L|\right)$ pseudo Lusin set. There will be pseudo Lusin sets even when there are no (classical) Lusin sets: If unif $\left(\left\langle J_{\mathbb{R}}\right\rangle\right)>\operatorname{add}\left(\left\langle J_{\mathbb{R}}\right\rangle, \subset\right)$ then every set of real numbers of cardinality $\aleph_{1}$ is meager, whence there is no Lusin set in the classical sense. Now let $\left\{M_{\alpha}: \alpha<\operatorname{add}\left(\left\langle J_{\mathbb{R}}\right\rangle, \subset\right)\right\}$ be a family of meager sets such that

1. $M_{\alpha} \subset M_{\beta}$ whenever $\alpha<\beta<\operatorname{add}\left(\left\langle J_{\mathbb{R}}\right\rangle, \subset\right)$ and

2. $\cup_{\alpha<\operatorname{add}\left(\left\langle J_{\mathbb{R}}\right\rangle, \subset\right)} M_{\alpha}$ is not meager.

Then the set $L=\left\{M_{\alpha}: \alpha<\operatorname{add}\left(\left\langle J_{\mathbb{R}}\right\rangle, \subset\right)\right\}$ is a $\left(\operatorname{add}\left(\left\langle J_{\mathbb{R}}\right\rangle, \subset\right), \operatorname{add}\left(\left\langle J_{\mathbb{R}}\right\rangle, \subset\right.\right.$ )) pseudo Lusin set.

It is also well known that these hypotheses on the ideal of meager subsets of the real line are consistent. For example, it is consistent that 
the real line is a union of $\aleph_{1}$ meager sets and that each set of real numbers of cardinality less than $\aleph_{2}$ is meager (see e.g. [M], $\S 6$ ).

The reader should also compare our notion of a $(\kappa, \lambda)$ - pseudo Lusin set with Cichon's notion of a $(\kappa, \lambda)$ - Lusin set (see [i]).

The connection between the irredundancy property and the existence of certain pseudo Lusin sets is given by the following proposition. The argument in its proof is well known in the special case when $P$ is the collection of countable subsets of some infinite set, ordered by set inclusion (see the proof of 4.4 on p. 409 of 迥).

Proposition 1 Let $(P,<)$ be a directed partially ordered set. Then the following statements are equivalent:

1. There is an $(\operatorname{add}(P,<), \operatorname{cof}(P,<))$ pseudo Lusin set for $(P,<)$,

2. $(P,<)$ has the irredundancy property,

3. There is a cofinal $(\operatorname{add}(P,<), \operatorname{cof}(P,<))$ pseudo Lusin set for $(P,<)$,

Proof. That 1. implies 2:

Let $L=\left\{x_{\xi}: \xi<\operatorname{cof}(P,<)\right\}$ be such a pseudo Lusin set and let $\left\{a_{\xi}\right.$ : $\xi<\operatorname{cof}(P,<)\}$ be a cofinal subfamily of $P$. For each $\xi<\operatorname{cof}(P,<)$ choose $z_{\xi} \in P$ such that $x_{\xi}, a_{\xi} \leq z_{\xi}$. Put $\mathcal{A}=\left\{z_{\xi}: \xi<\operatorname{cof}(P,<)\right\}$. Then $\mathcal{A}$ is an irredundant cofinal family.

That 2. implies 3:

Let $\mathcal{A}$ be an irredundant cofinal family. We may assume that the cardinality of this family is $\operatorname{cof}(P,<)$. Then $\mathcal{A}$ is an example of a cofinal $(\operatorname{add}(P,<), \operatorname{cof}(P,<))$ pseudo Lusin set.

It is clear that 3 . implies 1.

Corollary 2 Let $\kappa>\lambda \geq \aleph_{0}$ be cardinals, $\lambda$ regular. If $\operatorname{cof}\left([\kappa]^{<\lambda}, \subset\right)=$ $\kappa$, then $\left([\kappa]^{<\lambda}, \subset\right)$ has the irredundancy property.

Proof. Let $\left\{S_{\alpha}: \alpha<\kappa\right\}$ be a pairwise disjoint subcollection from $[\kappa]^{<\lambda}$. Then this family is a $(\lambda, \kappa)$ pseudo Lusin set for this ideal. Applying the cofinality hypothesis we conclude that this ideal has the irredundancy property.

The ideal of finite subsets of an infinite set has the irredundancy property; the set of one-element subsets of such an infinite set forms an appropriate pseudo Lusin set for this ideal.

Lemma 3 Let $\kappa>\lambda$ be an uncountable cardinal numbers, $\lambda$ regular. Then the following statements are equivalent:

1. The ideal $\left([\kappa]^{<\lambda}, \subset\right)$ has cofinality $\kappa$.

2. There is a free ideal $J$ such that:

(a) $\operatorname{add}(J, \subset)=\lambda$, 
(b) $\operatorname{cof}(J, \subset)=\kappa$ and

(c) $(J, \subset)$ has the irredundancy property.

Proof. The proof of $1 \Rightarrow 2$ is trivial. We show that 2 implies 1 . Let $J$ be a free ideal on the set $S$ such that $\operatorname{cof}(J, \subset)=\kappa$ and $\operatorname{add}(J, \subset$ )$=\lambda$, and $(J, \subset)$ has the irredundancy property. Let $L \subset J$ be an $(\lambda, \kappa)$ pseudo Lusin set for $J$. Also let $\mathcal{C} \subset J$ be a cofinal family of cardinality $\kappa$. For each $X \in \mathcal{C}$ define: $S_{X}=\{Y \in L: Y \subseteq X\}$. Then the collection $\mathcal{B}=\left\{S_{X}: X \in \mathcal{C}\right\}$ is cofinal in $\left([L]^{<\lambda}, \subset\right)$.

The following examples play an important role in our game-theoretic applications.

\section{Example 1: The ideal of countable subsets of an infinite set.}

Let $\kappa$ be an uncountable cardinal number. Then $\operatorname{add}\left([\kappa]^{\leq \aleph_{0}}, \subset\right)=\aleph_{1}$ and $b u\left([\kappa]^{\leq \aleph_{0}}, \subset\right) \geq \aleph_{1}$. For uncountable cardinal numbers $\kappa$ it is always the case that $\kappa \leq \operatorname{cof}\left([\kappa] \leq \aleph_{0}, \subset\right)$. A set of the form $\left\{\left\{\alpha_{\xi}\right\}: \xi<\kappa\right\}$ (where this enumeration is bijective and $\lambda \leq \kappa)$ is an $\left(\omega_{1}, \kappa\right)$ pseudo Lusin set for $[\kappa] \leq \aleph_{0}$. The only difficult cases to decide whether or not the irredundancy property is present are those where $\kappa<\operatorname{cof}\left([\kappa] \leq \aleph_{0}, \subset\right)$; this occurs for example when $\kappa$ has countable cofinality. It turns out that for these the irredundancy property is not decidable by the axioms of traditional set theory:

1. In To3], Todorčević shows that if for each uncountable cardinal $\lambda$ of countable cofinality the assertions

(a) $\left.\operatorname{cof}([\lambda]]^{\leq \aleph_{0}}, \subset\right)=\lambda^{+}$and

(b) $\square_{\lambda}$

are true, then for each uncountable cardinal number $\kappa$ there is a cofinal family $\mathcal{K} \subset[\kappa]^{\aleph_{0}}$ such that $|\{A \cap X: X \in \mathcal{K}\}| \leq \aleph_{0}$ for any countable subset $A$ of $\kappa$. Such a family $\mathcal{K}$ is an example of an $\left(\operatorname{add}\left([\kappa]^{\leq \aleph_{0}}, \subset\right), \operatorname{cof}\left([\kappa]^{\leq \kappa_{0}}, \subset\right)\right)$ pseudo Lusin set for $\left([\kappa]^{\leq \aleph_{0}}, \subset\right)$. These particular examples of pseudo Lusin sets are called cofinal Kurepa families. Thus it is true in the constructible universe, $\mathbf{L}$ that $\left([\kappa]^{<\aleph_{0}}, \subset\right)$ has the irredundancy property for each infinite $\kappa$.

2. One might ask if any hypotheses beyond $Z F C$ are necessary to obtain the conclusion that $\left([\kappa]^{\leq \aleph_{0}}, \subset\right)$ has the irredundancy property. Todorčević has shown in [To2] that for an infinite cardinal number $\kappa$ the following statements are equivalent:

(a) $b u\left([\kappa] \leq \aleph_{0}, \subset\right)=\aleph_{1}$.

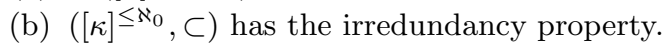

He also noted (p. 843 of [To4) that the version

$$
\left(\aleph_{\omega+1}, \aleph_{\omega}\right) \rightarrow\left(\omega_{1}, \omega\right)
$$

of Chang's Conjecture implies that $\aleph_{1}<b u\left(\left[\aleph_{\omega}\right]^{\leq \aleph_{0}}, \subset\right.$ ) (and thus this ideal does not have the irredundancy property). Now [L-M-S] established the consistency of the above version of Chang's Conjecture modulo the consistency of the existence of a fairly large cardinal. 
3. This takes care of uncountable cardinals of countable cofinality. What is the situation for those of uncountable cofinality? It is clear that $\left([\kappa]^{\leq \aleph_{0}}, \subset\right)$ has the irredundancy property if $\kappa$ is $\aleph_{n}$ for some finite $n$ or if, for some $m<\omega, \kappa$ is the $m$-th successor of a singular strong limit cardinal of uncountable cofinality. In fact, the axiomatic system of traditional set theory has to be strengthened fairly dramatically before one could create circumstances where there is a cardinal number of uncountable cofinality which is strictly less than the cofinality of its ideal of countable sets; it follows from Lemma 4.10 of $\mathrm{J}-\mathrm{M}-\mathrm{P}-\mathrm{S}$ ] that if there is a cardinal number of uncountable cofinality which is smaller than the cofinality of its ideal of countable sets, then there is an inner model with many measurable cardinal numbers.

Information about the ideal of countable subsets of some infinite set can be used to gain information about some other ideals, using the notion of a locally small family.

Definition 3 A family $\mathcal{F}$ of subsets of a set $S$ is locally small if:

$$
|\{Y \in \mathcal{F}: Y \subseteq X\}| \leq \aleph_{0}
$$

for each $X$ in $\mathcal{F}$.

If the ideal of countable subsets of an infinite set has an irredundant cofinal family then that cofinal family is ipso facto locally small. If there is an $\left(\omega_{1}, \operatorname{cof}(J, \subset)\right)$ pseudo Lusin set for the $\sigma$-complete free ideal $J$ on the set $S$, then $J$ contains a locally small cofinal family.

\section{Example 2: The ideal of meager subsets of the real line}

Assume that $\operatorname{add}\left(\left\langle J_{\mathbb{R}}\right\rangle, \subset\right)=\operatorname{cof}\left(\left\langle J_{\mathbb{R}}\right\rangle, \subset\right)$ (This equation is for example implied by Martin's Axiom). Then $\left\langle J_{\mathbb{R}}\right\rangle$ has the irredundancy property. In this case one may insure that the cofinal family which witnesses the irredundancy is a well-ordered chain of meager sets. By the results cited from $[\mathrm{M}]$ and $[\mathrm{F}]$, the hypothesis implies that $\mathfrak{b}=\mathfrak{d}$. It is well known that the reverse implication is not provable.

Irredundancy does not require having a well ordered cofinal chain of meager sets. For let an initial ordinal be given. According to a theorem of Kunen ([K], p. 906, Theorem 3.18) it is consistent that the cardinality of the real line is regular and larger than that initial ordinal, and at the same time there is an $\left(\omega_{1}, \mathfrak{c}\right)$ pseudo Lusin set. It follows that $\left\langle J_{\mathbb{R}}\right\rangle$ has a locally small cofinal family of cardinality $\mathfrak{c}$. In particular, $\left\langle J_{\mathbb{R}}\right\rangle$ has the irredundancy property. If the continuum is larger than $\aleph_{1}$ it also follows that this ideal has no cofinal well-ordered chain.

Stevo Todorčević has informed us that it is also consistent, modulo the consistency of a form of Chang's Conjecture that $\left\langle J_{\mathbb{R}}\right\rangle$ does not have the irredundancy property. Actually, something apparently weaker than that form of Chang's Conjecture is used: we present this result of Todorčević's in Theorem 4, which he kindly permitted us to include in this paper. 
Theorem 4 (Todorčević) If " $\mathrm{ZFC}+M A_{\aleph_{1}}+$ there is no Kurepa family in $\left[\aleph_{\omega}\right]^{\aleph_{0}}$ of cardinality larger than $\aleph_{\omega}$ " is a consistent theory, then so is the theory "ZFC + bu $\left(\left\langle J_{\mathbb{R}}\right\rangle, \subset\right)>\operatorname{add}\left(\left\langle J_{\mathbb{R}}\right\rangle, \subset\right)=\aleph_{1}$ ".

Proof Let $\mathbf{P}$ be the set of finite functions with domain a subset of $\aleph_{\omega}$ and range a subset of $\omega$ (in other words, $\mathbf{P}$ is the standard set for adding $\aleph_{\omega}$ Cohen reals). For $p$ and $q$ in $\mathbf{P}$ we write $p<q$ if $q \subset p$. For $D$ a countable subset of $\aleph_{\omega}$ we write $\mathbf{P}(D)$ for the set of elements of $\mathbf{P}$ whose domains are subsets of $D$.

Suppose we have a sequence $\left\{N_{\xi}: \xi<\theta\right\}\left(\theta>\aleph_{\omega}\right)$ of $\mathbf{P}$-names for meager sets of reals. Let $D_{\xi} \in\left[\aleph_{\omega}\right]^{\aleph_{0}}$ be the support of $N_{\xi}$ i.e., $N_{\xi} \in \mathbf{V}^{\mathbf{P}\left(D_{\xi}\right)}$. By the hypothesis of the theorem and by Theorem 1 of To3] there is an uncountable set $A \subset \theta$ such that $D=\cup_{\xi \in A} D_{\xi}$ is countable. Thus, $N_{\xi} \in \mathbf{V}^{\mathbf{P}(D)}$ for each $\xi \in A$. Since $\mathbf{P}(D)$ is essentially the poset for adding one Cohen real and since $M A_{\aleph_{1}}$ holds, $\mathbf{V}^{\mathbf{P}(D)} \models " \cup_{\xi \in A} N_{\xi}$ is meager" (because $\mathbf{V}^{\mathbf{P}(D)} \models " M A(\sigma-$ centered)").

The hypothesis of Theorem is consistent modulo the consistency of the relevant form of Chang's Conjecture, because that form of the conjecture is preserved by c.c.c. generic extensions.

\section{The coherent decomposition property}

Let $J$ be a free ideal on a set $S$ and let $\langle J\rangle$ be its $\sigma$-completion. Let $\mathcal{A}$ be a subcollection of $\langle J\rangle$.

Definition 4 1. $\mathcal{A}$ has a coherent decomposition if there is for each $A \in \mathcal{A}$ a sequence $\left(A^{n}: n<\omega\right)$ such that:

(a) $A^{n} \in J$ for each $n$,

(b) $A^{n} \subseteq A^{m}$ whenever $n<m<\omega$, and

(c) For all $A$ and $B$ in $\mathcal{A}$ such that $A \subset B$, there is an $m$ such that $A^{n} \subseteq B^{n}$ whenever $n \geq m$.

The collection $\left\{\left(A^{n}: n<\omega\right): A \in \mathcal{A}\right\}$ is said to be a coherent decomposition for $\mathcal{A}$.

2. The ideal $J$ has the coherent decomposition property if some cofinal subset of $\langle J\rangle$ has a coherent decomposition.

It is worth mentioning that if $J$ has the coherent decomposition property and if $\langle J\rangle$ has a cofinal chain, than the family $\langle J\rangle$ itself has a coherent decomposition. We now explore the coherent decomposition property for our examples.

\section{Example 1: (continued)}

Theorem 5 Let $\mathcal{A}$ be a locally small family of countable sets such that $(\mathcal{A}, \subset)$ is a well-founded partially ordered set. Then $\mathcal{A}$ has a coherent decomposition. 
Proof. Let $\Phi: \mathcal{A} \rightarrow \alpha$ be a function to an ordinal $\alpha$ such that $\Phi(A)<$ $\Phi(B)$ for all $A \subset B$ in $\mathcal{A}$ (i.e., a rank function). Since $\mathcal{A}$ is locally small we may assume that $\alpha$ is $\omega_{1}$.

For $A$ in $\mathcal{A}$ with $\Phi(A)=0$, choose a sequence $\left(A^{n}: n<\omega\right)$ of finite subsets of $A$ such that $A=\cup_{n<\omega} A^{n}$ and $A^{n} \subseteq A^{n+1}$ for all n.

Let $0<\beta<\omega_{1}$ be given and assume that we have already assigned to each $A$ in $\mathcal{A}$ for which $\Phi(A)<\beta$, a sequence $\left(A^{n}: n<\omega\right)$ in compliance with 1 and 2. Now Let $B$ be an element of $\mathcal{A}$ such that $\Phi(B)=\beta$. Write $F(B)=\{A \in \mathcal{A}: A \subset B\}$.

To begin, arbitrarily choose a sequence $\left(S_{n}: n<\omega\right)$ of finite sets such that $B=\cup_{n<\omega} S_{n}$. For each $A \in F(B)$, define $g_{A}: \omega \rightarrow \omega$ such that for each $n<\omega$,

$$
g_{A}(n)=\min \left\{k<\omega: A^{n} \subseteq S_{0} \cup \ldots \cup S_{k}\right\} .
$$

Then $\left\{g_{A}: A \in F(B)\right\}$ is countable since $\mathcal{A}$ is locally small. Let $f \in$ ${ }^{\omega} \omega$ be a strictly increasing function such that $g_{A} \ll f$ for each $A$ in $F(B)$. Define:

$$
B^{n}=S_{0} \cup \ldots \cup S_{f(n)}
$$

for each $\mathrm{n}$. Then $\left(B^{n}: n<\omega\right)$ is as required.

Corollary 6 Let $J$ be a free ideal on a set $S$ and let $\mathcal{A}$ be a locally small family of sets in $\langle J\rangle$ such that $(\mathcal{A}, \subset)$ is a well-founded partially ordered set. Then $\mathcal{A}$ has a coherent decomposition.

Proof. For each $B$ in $\mathcal{A}$, let $\left(S_{n}(B): n<\omega\right)$ be a sequence from $J$ such that $B=\cup_{n<\omega} S_{n}(B)$. Also write $\Gamma(B)=\{A \in \mathcal{A}: A \subseteq B\}$. Then $\mathcal{B}=\{\Gamma(A): A \in \mathcal{A}\}$ is a well-founded, locally small collection of countable subsets of $\mathcal{A}$. Choose, by Theorem 5 , for each $A \in \mathcal{A}$ a sequence $\left(\Gamma(A)^{n}: n<\omega\right)$ of finite subsets of $\Gamma(A)$ such that:

1. $\Gamma(A)=\cup_{n<\omega} \Gamma(A)^{n}$ where $\Gamma(A)^{n} \subseteq \Gamma(A)^{n+1}$ for each $\mathrm{n}$, and

2. for all $A$ and $B$ in $\mathcal{A}$ with $A \subset B$ there exists an $m$ such that:

$$
\Gamma(A)^{n} \subseteq \Gamma(B)^{n}
$$

for all $n \geq m$.

For each $A$ in $\mathcal{A}$ and each $n<\omega$ define:

$$
A^{n}=\cup\left\{S_{j}(B): j \leq n \text { and } B \in \Gamma(A)^{n}\right\} .
$$

Then the sequences $\left(A^{n}: n<\omega\right)$ are as required.

Corollary 7 If $\left([\kappa]^{\leq \aleph_{0}}, \subset\right)$ has the irredundancy property, then it has the coherent decomposition property.

Proof An irredundant cofinal family is necessarily locally small. We may thin out any cofinal family to a well-founded cofinal family. Now apply Theorem 


\section{Example 2: (continued)}

We show that the ideal of meager sets of the real line has the coherent decomposition property, and also that it has a second combinatorial property which plays an important role in our game-theoretic applications. It is convenient, for this section, to work with the set ${ }^{\omega} 2$, with the usual Tychonoff product topology $(2=\{0,1\}$ is taken to have the discrete topology) in place of $\mathbb{R}$. For a subset $S$ of the domain of a function $g$, the symbol $g\left\lceil_{S}\right.$ denotes the restriction of $g$ to the set $S$. For $s$ an element of ${ }^{<\omega_{2}} 2$, the symbol $[s]$ denotes the set of all those $x$ in ${ }^{\omega} 2$ for which $x \Gamma_{\text {length }(s)}=s$. Subsets of ${ }^{\omega_{2}}$ of the form $[s]$ where $s$ ranges over ${ }^{<\omega_{2}} 2$, form a base for the topology of ${ }^{\omega} 2$. Let $f \in{ }^{\omega} \omega$ be a strictly increasing sequence and let $x$ be an element of ${ }^{\omega} 2$. Define:

$$
B_{x, f}=\left\{z \in{ }^{\omega} 2: \forall_{n}^{\infty}\left(z\left\lceil_{[f(n), f(n+1))} \neq x\left\lceil_{[f(n), f(n+1))}\right)\right\} .\right.\right.
$$

Now also fix an $n \in \omega$ and define

$$
B_{x, f}^{n}=\left\{z \in{ }^{\omega} 2:(\forall k \geq n)\left(z\left\lceil_{[f(k), f(k+1))} \neq x\left\lceil_{[f(k), f(k+1))}\right)\right\} .\right.\right.
$$

Then $B_{x, f}^{m} \subseteq B_{x, f}^{n}$ whenever $m<n<\omega$; also, $B_{x, f}=\cup_{n<\omega} B_{x, f}^{n}$.

Proposition 8 For $x, y \in{ }^{\omega} 2$ and strictly increasing $f, g \in{ }^{\omega} \omega$, the following assertions are equivalent:

1. $B_{x, f} \subset B_{y, g}$.

2. (a) $B_{x, f} \neq B_{y, g}$ and

(b) $\left(\forall_{n}^{\infty}\right)(\exists k)\left(g(n) \leq f(k)<f(k+1) \leq g(n+1)\right.$ and $x\left\lceil_{[f(k), f(k+1))}=\right.$ $y\lceil[f(k), f(k+1)))$

Proof. That 1 implies 2 requires some thought:

If 1 holds, then (a) of 2 holds. Assume the negation of $2(\mathrm{~b})$. It reads:

$$
\left(\exists_{n}^{\infty}\right)(\forall k)\left(\neg ( g ( n ) \leq f ( k ) < f ( k + 1 ) \leq g ( n + 1 ) ) \text { or } \neg \left(x\left\lceil_{[f(k), f(k+1))}=y\left\lceil_{[f(k), f(k+1))}\right)\right)\right.\right.
$$

Put $S=\left\{n<\omega:(\forall k)\left(\neg([f(k), f(k+1)] \subseteq[g(n), g(n+1)])\right.\right.$ or $\neg\left(x\left\lceil_{[f(k), f(k+1))}=\right.\right.$ $y\lceil[f(k), f(k+1)))\}$. Our hypothesis is that $S$ is an infinite set.

Consider an $n$ in $S$. For each $k$, there are the following possibilities:

1. $\neg([f(k), f(k+1)] \subseteq[g(n), g(n+1)]$

2. $[f(k), f(k+1)] \subseteq[g(n), g(n+1)]$, but $x\left\lceil_{[f(k), f(k+1))} \neq y\left\lceil_{[f(k), f(k+1))}\right.\right.$.

Put $S_{n}=\{k: 2$ holds for $k\}$. We consider two cases.

Case 1: There are infinitely many $n$ for which $S_{n}$ is nonempty. Choose an infinite sequence $\left(n_{1}, n_{2}, n_{3}, \ldots\right)$ from $S$ such that:

1. $S_{n_{m}} \neq \emptyset$,

2. $n_{m+1}>g\left(n_{m}+1\right)$, and

3. $(\exists k)\left(g\left(n_{m}+1\right)<f(k)<g\left(n_{m+1}\right)\right)$, for each $m$, and 
4. $f(1)<g\left(n_{1}\right)$.

This is possible because $f$ and $g$ are increasing, and $S$ is infinite. Put $T=\cup_{j=1}^{\infty}\left[g\left(n_{j}\right), g\left(n_{j}+1\right)\right)$. Define $z$, an element of ${ }^{\omega} 2$, so that $z\left\lceil_{T}=y\left\lceil_{T}\right.\right.$ and $z(n)=1-x(n)$ for each $n \in \omega \backslash T$. Then $z \in B_{x, f}$ while $z \notin B_{y, g}$. Thus 1 fails in this case.

Case 2: There are only finitely many $n \in S$ for which $S_{n}$ is nonempty.

We may assume that $S_{n}=\emptyset$ for each $n \in S$. Consider $n \in S$. We then have that for each $k \in \omega,[f(k), f(k+1)) \nsubseteq[g(n), g(n+1))$. We distinguish between two possibilities:

1. $(\exists k)(g(n) \leq f(k)<g(n+1))$ or

2. $(\forall k)(f(k) \notin[g(n), g(n+1))$

Case 2 (A): Possibility 1 occurs for infinitely many $n \in S$ :

Choose $n_{1}<n_{2}<n_{3}<\ldots$ from $S$ such that

- $2 \cdot n_{j} \leq n_{j+1}$ for each $j$,

- for each $j$ there is a $k$ such that $g\left(n_{j}+1\right)<f(k)<g\left(n_{j+1}\right)$,

- for each $j$ there is a $k$ such that $f(k) \in\left[g\left(n_{j}\right), g\left(n_{j+1}\right)\right)$, and

- $f(1)<g\left(n_{1}\right)$.

Put $T=\cup_{j=1}^{\infty}\left[g\left(n_{j}\right), g\left(n_{j}+1\right)\right)$ and define $z$ so that $z\left\lceil_{T}=y\left\lceil_{T}\right.\right.$, and $z(n)=1-x(n)$ for each $n \in \omega \backslash T$. From the hypothesis of Case 2(A) it follows that $z \in B_{x, f}$, but $z \notin B_{y, g}$. Thus, 1 of the Proposition fails also in this case.

Case 2 (B): Possibility 1 occurs for only finitely many $n \in S$ :

We may assume that possibility 2 occurs for each $n \in S$. Choose $k_{1}<k_{2}<k_{3}<\ldots$ such that for each $j$ there is an $n \in S$ with $[g(n), g(n+1)) \subset\left[f\left(k_{j}\right), f\left(k_{j}+1\right)\right)$. For each $j$ choose $n_{j} \in S$ such that $\left[g\left(n_{j}\right), g\left(n_{j}+1\right)\right) \subset\left[f\left(k_{j}\right), f\left(k_{j}+1\right)\right)$. As before define $T=\cup_{j=1}^{\infty}\left[g\left(n_{j}\right), g\left(n_{j}+1\right)\right)$. Finally, define $z$ so that $z\left\lceil_{T}=y\left\lceil_{T}\right.\right.$ and $z(n)=1-x(n)$ for each $n \in \omega \backslash T$. Then $z \in B_{x, f}$ and $z \notin B_{y, g}$, showing that 1 of the Proposition fails also in this case.

This completes the proof of the Proposition.

Lemma 9 Let $f$ and $g$ be strictly increasing elements of ${ }^{\omega} \omega$ for which there is some $k<\omega$ such that $g(n+k)=f(n)$ for all but finitely many $n$. If $B_{x, f} \subseteq B_{y, g}$, then $B_{x, f}=B_{y, g}$.

Proof. Assume that $B_{x, f} \neq B_{y, g}$ and suppose that $B_{y, g} \not B_{x, f}$. We show that $B_{x, f} \not \subset B_{y, g}$. Let $z$ be an element of $B_{y, g} \backslash B_{x, f}$. Fix $N$ such that 
1. $z\left\lceil_{[g(n+k), g(n+k+1))} \neq y\left\lceil_{[g(n+k), g(n+k+1))}\right.\right.$ and

2. $f(n)=g(n+k)$

for each $n \geq N$.

Since $z$ is not an element of $B_{x, f}$, there are infinitely many $n \geq$ $N$ for which $z\left\lceil_{[f(n), f(n+1))}=x\left\lceil_{f(n), f(n+1))}\right.\right.$. Consequently the set $S=\left\{n \geq N: x\left\lceil_{[f(n), f(n+1))} \neq y\left\lceil_{[f(n), f(n+1))}\right\}\right.\right.$ is infinite. Now define $t$ such that $t\left\lceil_{[f(n), f(n+1))}=y\left\lceil_{[f(n), f(n+1))}\right.\right.$ for each $n \in S$, and $t(m)=1-x(m)$ for each $m \in \omega \backslash\left(\cup_{n \in S}[f(n), f(n+1))\right)$. Then $t$ is in $B_{x, f}$ but not in $B_{y, g}$.

Under the hypothesis of Lemma $9, x(n)=y(n)$ for all but finitely many $n$.

Proposition 10 Let $x, y$ be elements of ${ }^{\omega} 2$ and let $f, g$ be increasing elements of ${ }^{\omega} \omega$. Of the following two assertions, 1 implies 2 .

1. $B_{x, f} \subset B_{y, g}$.

2. $f \ll g$.

Proof. Assume that $B_{x, f} \subset B_{y, g}$. Fix, by Proposition 8, an $N$ such that

$(\forall n \geq N)(\exists k)\left([f(k), f(k+1)] \subseteq[g(n), g(n+1)]\right.$ and $x\left\lceil_{[f(k), f(k+1))}=\right.$ $y\lceil[f(k), f(k+1)))$.

For each $n \geq N$ choose $k_{n}$ such that $\left[f\left(k_{n}\right), f\left(k_{n}+1\right)\right] \subseteq[g(n), g(n+$ 1)]. It follows that $k_{n}+1 \leq k_{n+1}$ for each $n \geq N$ (since $f$ and $g$ are increasing).

Claim: $\left[f\left(k_{n}\right), f\left(k_{n}+1\right)\right] \subset[g(n), g(n+1)]$ for infinitely many $\mathrm{n}$. Proof of the claim: For otherwise, fix $M \geq N$ such that $\left[f\left(k_{n}\right), f\left(k_{n+1}\right]=\right.$ $[g(n), g(n+1)]$ for each $n \geq M$. Then we have $k_{n+1}=k_{n}+1$ for each $n \geq M$. It follows that $g(n)=f\left(n+\left(k_{M}-M\right)\right)$ for all $n \geq M$. Then Lemma 9 implies that $B_{x, f}=B_{y, g}$, contrary to the fact that $B_{x, f}$ is a proper subset of $B_{y, g}$. This completes the proof of the claim.

Thus, there are infinitely many $n$ for which $k_{n+1}>k_{n}+1$. Let $m>1$ be given, and fix $L \geq M$ such that $\left|\left\{n<L: k_{n+1}>k_{n}+1\right\}\right| \geq$ $k_{1}+m$. Then $k_{n}>(n+m)$ for each $n \geq L$; we have

$$
f(n+1)<f(n+m) \leq f\left(k_{n}\right)<g(n+1)
$$

for each $n \geq L$. In particular, $m \leq g(n+1)-f(n+1)$ for each $n \geq L$. This completes the proof that $f \ll g$.

Proposition 11 Let $x$ and $y$ be elements of ${ }^{\omega} 2$ and let $f$ and $g$ be increasing elements of ${ }^{\omega} \omega$. If $B_{x, f} \subset B_{y, g}$, then there is an $m<\omega$ such that $B_{x, f}^{n} \subseteq B_{y, g}^{n}$ whenever $n \geq m$. 
Proof From our hypotheses and Proposition 8 there is an $m$ such that for each $n \geq m$ there is a $k$ such that $[f(k), f(k+1)) \subseteq[g(n), g(n+1))$ and $x\left\lceil_{[f(k), f(k+1))}=y\left\lceil_{[f(k), f(k+1))}\right.\right.$. By Proposition 10 there is an $M>m$ such that $f(j) \leq g(j)$ for each $j \geq M$. We show that $B_{x, f}^{n} \subseteq B_{y, g}^{n}$ for each $n \geq M$.

Let $z$ be an element of $B_{x, f}^{n}$. Then $z\left\lceil_{[f(j), f(j+1))} \neq x\left\lceil_{[f(j), f(j+1))}\right.\right.$ for each $j \geq n$. But consider any $j \geq \mathrm{n}$. Then there is a $k$ such that $[f(k), f(k+1)) \subset[g(j), g(j+1)) ; k \geq j$ for any such $k$, by the choice of $M$. It follows that $z\left\lceil_{[g(j), g(j+1))} \neq y\left\lceil_{[g(j), g(j+1))}\right.\right.$. Thus, $z$ is also an element of $B_{y, g}^{n}$.

Proposition 12 For each $X \in\left\langle J_{\mathbb{R}}\right\rangle$ there are an $x$ in ${ }^{\omega} 2$ and an increasing $f$ in ${ }^{\omega} \omega$ such that $X \subset B_{x, f}$.

Proof. Let $X$ be a meager set. We may assume that $X=\cup_{n=0}^{\infty} X_{n}$ where $X_{n} \subseteq X_{n+1}$ and $X_{n}$ is closed, nowhere dense for each $n$. Fix a wellordering of ${ }^{<\omega} 2$, and define $\left(s_{n}: n<\omega\right)$ and $f$ in ${ }^{\omega} \omega$ as follows:

Take $s_{0}=\emptyset$ and $f(0)=0$. Assume that $s_{1}, s_{2}, \ldots, s_{n}$ and $f(1), \ldots, f(n)$ have been defined so that:

1. $s_{1}$ is the first element of ${ }^{<\omega} 2$ such that $\left[s_{1}\right] \cap X_{1}=\emptyset$ and $f(1)=$ length $\left(s_{1}\right)$,

2. $s_{j+1}$ is the first element of $<\omega^{2}$ such that $\left[t^{\frown} s_{j+1}\right] \cap X_{j}=\emptyset$ for each $t$ in ${ }^{\leq f(j)} 2$, and $f(j+1)=\sum_{i=0}^{j+1}$ length $\left(s_{i}\right)$ for each $j<n$.

Then let $s_{n+1}$ be the first element of $<\omega_{2}$ such that $\left[t^{\frown} s_{n+1}\right] \cap X_{n}=\emptyset$ for each $t$ in $\leq^{(n)} 2$; put $f(n+1)=f(n)+$ length $\left(s_{n+1}\right)$.

Finally, set $x=s_{1} s_{2} s_{3} \ldots$...

Claim: $X \subseteq B_{x, f}$.

For suppose that $z$ is not an element of $B_{x, f}$. Then there are infinitely many $n$ for which $z\left\lceil_{[f(n), f(n+1))}=x\left\lceil_{[f(n), f(n+1))}\right.\right.$; in other words, there are infinitely many $n$ for which $z\left\lceil[f(n), f(n+1))=s_{n+1}\right.$. Now fix an $m$. Choose an $n>m$ such that $z\left\lceil_{[f(n), f(n+1))}=s_{n+1}\right.$. From the choice of $s_{n+1}$ it follows that $\left[z[f(n+1)] \cap X_{m}=\emptyset\right.$; in particular, $z \notin X_{m}$. Consequently, $z$ is not an element of $X$.

Proposition 13 Each $B_{x, f}^{n}$ is in $J_{\mathbb{R}}$.

Proof. Consider an $s$ from ${ }^{\omega} 2$ for which $[s] \cap B_{x, f}^{n} \neq \emptyset$. Choose $m$ such that $f(m)>$ length $(s)$ and $m>n$. Then choose $t$ from ${ }^{<} \omega_{2}$ such that length $(s \frown t) \geq f(m+1)$ and $s \frown t\left\lceil_{[f(m), f(m+1))}=\right.$ $x\left\lceil_{[f(m), f(m+1))}\right.$. Then $[s \frown t] \cap B_{x, f}^{n}=\emptyset$. It follows that $B_{x, f}^{n}$ is nowhere dense.

Consequently, $B_{x, f}$ is a meager set for each $x$ in ${ }^{\omega_{2}} 2$ and for each increasing $f$ from ${ }^{\omega} \omega$.

Theorem $14\left\langle J_{\mathbb{R}}\right\rangle$ has a cofinal family which embeds in $\left({ }^{\omega} \omega, \ll\right)$ and which has the coherent decomposition property. 
Proof. By Propositions 13 and 12 the family of sets of the form $B_{x, f}$ where $f$ is an increasing element of ${ }^{\omega} \omega$ and $x$ is an element of ${ }^{\omega} 2$, is a cofinal family of meager sets. By Proposition 11, this family has the coherent decomposition property. Also, the mapping which assigns $f$ to $B_{x, f}$ is, according to Proposition 10, an order preserving mapping.

\section{Example 3: Cardinals of countable cofinality}

Here is a result which is quite analogous to Theorem .

Theorem 15 Let $\lambda$ be an uncountable cardinal number which has countable cofinality. Let $\lambda_{0}<\lambda_{1}<\ldots$ be a sequence of infinite regular cardinal numbers which converges to $\lambda$. Let $(\mathcal{A}, \subset)$ be a well-founded family of sets, each of cardinality $\lambda$, such that

$$
|\{Y \in \mathcal{A}: Y \subseteq X\}| \leq \lambda
$$

for each $X$ in $\mathcal{A}$. Then $\mathcal{A}$ has the coherent decomposition property. In particular:

There exists for each $A \in \mathcal{A}$ a sequence $\left(A^{n}: n<\omega\right)$ such that:

1. $\left|A^{n}\right| \leq \lambda_{n}$ for all $n$,

2. $A^{n} \subseteq A^{n+1}$ for all $n$,

3. $A=\cup_{n=0}^{\infty} A^{n}$ and

4. if $A \subset B$, then there is an $m<\omega$ such that $A^{n} \subseteq B^{n}$ for all $n \geq m$.

Proof. Let $\Phi: \mathcal{A} \rightarrow \lambda^{+}$be a rank function. For all $A$ in $\mathcal{A}$ with $\Phi(A)=0$, choose $\left(A^{n}: n<\omega\right)$ arbitrary, subject only to 1,2 and 3 .

Let $0<\gamma<\lambda^{+}$be given and assume that $\left(A^{n}: n<\omega\right)$ has been assigned to each $A$ from $\mathcal{A}$ for which $\Phi(A)<\gamma$, in such a way that 1, 2, 3 and 4 are satisfied. Consider $B$ in $\mathcal{A}$ with $\Phi(B)=\gamma$. Write $F(B)$ for $\{A \in \mathcal{A}: A \subseteq B\}$ and write $F(B)=\cup_{n=0}^{\infty} F_{n}(B)$ where

1. $F_{0}(B) \subseteq F_{1}(B) \subseteq \ldots$, and

2. $\left|F_{n}(B)\right| \leq \lambda_{n}$ for all $n$.

Also let $B=\cup_{n=0}^{\infty} X_{n}$ where $X_{0} \subseteq X_{1} \subseteq \ldots$ and $X_{n} \leq \lambda_{n}$ for all $n$. Finally put $B^{n}=\left(\cup\left\{A^{n}: A \in F_{n}(B)\right\}\right) \cup X_{n}$ for each $n$. Then $\left(B^{n}: n<\omega\right)$ is as required.

Corollary 16 Let $\lambda$ be a cardinal number of countable cofinality. If $([\kappa] \leq \lambda, \subset)$ has the irredundancy property then it has the coherent decomposition property. 


\section{Applications}

The $\omega$-path partition relation is the one other combinatorial ingredient in our technique for constructing winning $k$-tactics, or for defeating a given $k$-tactic for TWO. For a positive integer $n$, infinite cardinal number $\lambda$ and a partially ordered set $(P,<)$, the symbol

$$
(P,<) \rightarrow(\omega-\text { path })_{\lambda /<\omega}^{n}
$$

means that for every function $F:[P]^{n} \rightarrow \lambda$ there is an increasing $\omega$ sequence

$$
p_{1}<p_{2}<\ldots<p_{m}<\ldots
$$

such that the set $\left\{F\left(\left\{p_{j+1}, \ldots, p_{j+n}\right\}\right): j<\omega\right\}$ is finite. The negation of this assertion is denoted by the symbol

$$
(P,<) \not \rightarrow(\omega-\text { path })_{\lambda /<\omega}^{n} .
$$

This partition relation has been studied in S2. The reader should consult this reference about the various facts concerning the $\omega$-path relation which are used in the sequel.

\subsection{The game $M G(\mathcal{A}, J)$}

For a free ideal $J$ on an infinite set $S$ and for a family $\mathcal{A}$ in $\langle J\rangle$ with the property that for each $X \in \mathcal{A}$ there is a $Y \in \mathcal{A}$ such that $X \subset Y$, the game $M G(\mathcal{A}, J)$ is defined so that an $\omega$-sequence $\left(O_{1}, T_{1}, \ldots, O_{n}, T_{n}, \ldots\right)$ is a play if for each $n$,

1. $O_{n} \in \mathcal{A}$ is player ONE's move in inning $n$,

2. $T_{n} \in J$ is player TWO's move in inning $n$, and

3. $O_{n} \subset O_{n+1}$.

Player TWO wins this play if $\cup_{n=1}^{\infty} O_{n} \subseteq \cup_{n=1}^{\infty} T_{n}$.

Theorem 17 Let $J$ be a free ideal on a set $S$. If $\mathcal{A}$ is a family of sets in $\langle J\rangle$ such that:

1. for each $X \in \mathcal{A}$ there is a $Y \in \mathcal{A}$ such that $X \subset Y$,

2. $(\mathcal{A}, \subset) \nrightarrow \rightarrow(\omega-\text { path })_{\omega /<\omega}^{k}$ for some $k \geq 2$, and

3. $\mathcal{A}$ has a coherent decomposition

then TWO has a winning k-tactic in $M G(\mathcal{A}, J)$.

Proof. Choose a function $F:[\mathcal{A}]^{k} \rightarrow \omega$ which witnesses hypothesis 2. Also associate with each $A$ in $\mathcal{A}$ a sequence $\left(A^{n}: n<\omega\right)$ such that hypothesis 3 is satisfied.

Define a $k$-tactic, $\Upsilon$ for TWO as follows. Let $\left(X_{1}, \ldots, X_{j}\right)$ be given such that $j \leq k, X_{1} \subset \ldots \subset X_{j}$ and $X_{i} \in \mathcal{A}$ for $i \leq j$.

1. If $j<k$ : Then put $\Upsilon\left(X_{1}, \ldots, X_{j}\right)=X_{1}^{1} \cup \ldots \cup X_{j}^{1}$.

2. If $j=k$ : Let $m$ be such that 
- $m \geq F\left(\left\{X_{1}, \ldots, X_{k}\right\}\right)$ and

- $X_{1}^{n} \subseteq \ldots \subseteq X_{k}^{n}$ for all $n \geq m$.

Put $\Upsilon\left(X_{1}, \ldots, X_{k}\right)=X_{1}^{m} \cup \ldots \cup X_{k}^{m}$.

Then $\Upsilon$ is a winning $k$-tactic for TWO. For let $\left(O_{1}, T_{1}, \ldots, O_{n}, T_{n}, \ldots\right)$ be a play of $M G(\mathcal{A}, J)$ where:

- $T_{j}=\Upsilon\left(O_{1}, \ldots, O_{j}\right)$ for each $j \leq k$

- $T_{n+k}=\Upsilon\left(O_{n+1}, \ldots, O_{n+k}\right)$ for each $n<\omega$.

For each $t \geq 1$ let $m_{t}$ be the number associated with $\left(O_{t}, \ldots, O_{t+k-1}\right)$ in part 2 of the definition of $\Upsilon$. By the properties of $F$, the set $\left\{m_{t}: t=1,2,3, \ldots\right\}$ is infinite. Thus choose $t_{1}<t_{2}<\ldots$ such that $m_{j}<m_{t_{r}}$ for all $j<t_{r}$. It follows from the criteria used in the choices of the numbers $m_{t}$ that

$$
O_{1}^{m_{t_{r}}} \subseteq \ldots \subseteq O_{m_{t_{r}}}^{m_{t_{r}}}
$$

for all $r$. But $O_{m_{t_{r}}}^{m_{t_{r}}} \subseteq T_{m_{t_{r}}}$ for all $r$, according to the definition of $\Upsilon$. It follows that $\cup_{n=1}^{\infty} O_{n} \subseteq \cup_{n=1}^{\infty} T_{n}$.

Corollary 18 There is a cofinal family $\mathcal{A} \subset\left\langle J_{\mathbb{R}}\right\rangle$ such that TWO has a winning 2-tactic in $M G\left(\mathcal{A}, J_{\mathbb{R}}\right)$.

Proof. Let $\mathcal{A}$ be the family of meager sets provided by Theorem 14 . Thus, there is an order preserving function from $(\mathcal{A}, \subset)$ to $\left({ }^{\omega} \omega, \ll\right)$. But then $(\mathcal{A}, \subset) \not \rightarrow(\omega-\text { path })_{\omega /<\omega}^{2}$ holds, since $\left({ }^{\omega} \omega, \ll\right) \not \rightarrow(\omega-$ path $)_{\omega /<\omega}^{2}$ holds. By Theorem 14 the family $\mathcal{A}$ also satisfies the third hypothesis of Theorem 17 .

Corollary 19 Let $J$ be a free ideal on an infinite set. If $\mathcal{A}$ is a family of sets in $\langle J\rangle$ such that:

1. $\mathcal{A}$ is locally small,

2. for each $X \in \mathcal{A}$ there is a $Y \in \mathcal{A}$ such that $X \subset Y$, and

3. $(\mathcal{A}, \subset)$ is well-founded,

then TWO has a winning 2-tactic in $M G(\mathcal{A}, J)$.

Proof. The proof is analogous to that of Corollary 18; now we refer to the proof of Theorem $\mathrm{f}$, we observe that $\omega_{1} \leq \mathfrak{b}$, and invoke Theorem 17.

Corollary 20 Let $\lambda \leq \kappa$ be infinite cardinal numbers such that:

1. $\lambda$ has countable cofinality,

2. $\lambda^{+} \nrightarrow(\omega-\text { path })_{\omega /<\omega}^{2}$, and

3. $[\kappa] \leq \lambda$ has the irredundancy property. 
Then there is a cofinal family $\mathcal{A} \subset[\kappa]^{\lambda}$ such that TWO has a winning 2-tactic in $M G\left(\mathcal{A},[\kappa]^{<\lambda}\right)$.

Proof. Let $\mathcal{A}$ be a well-founded cofinal family in $[\kappa]^{\lambda}$ which is irredundant. Since there is a rank-function from $\mathcal{A}$ to $\lambda^{+}$it follows from hypothesis 2 that $(\mathcal{A}, \subset) \not \rightarrow(\omega-\text { path })_{\omega /<\omega}^{2}$. From Corollary 16 it follows that $\mathcal{A}$ also satisfies the third hypothesis of Theorem 17. By that theorem TWO then has a winning 2-tactic in the game $M G\left(\mathcal{A},[\kappa]^{<\lambda}\right)$.

Theorem 21 shows that under certain circumstances there is for each $n$ a free ideal $J_{n}$ and a cofinal family $\mathcal{A}_{n} \subset\left\langle J_{n}\right\rangle$ such that TWO does not have a winning $n$-tactic, but does have a winning $n+1$-tactic in $M G\left(\mathcal{A}_{n}, J_{n}\right)$. We think that Theorem 21 indicates some relevance of the games as considered here for Telgarsky's Conjecture (see 3.4).

Theorem 21 Let $\lambda$ be an infinite cardinal number. If there is a linearly ordered set $(L,<)$ such that:

1. $\operatorname{cof}(L,<)>\omega$,

2. $(L,<) \rightarrow(\omega-\text { path })_{\lambda /<\omega}^{2}$, but

3. $(L,<) \nrightarrow \rightarrow(\omega-\text { path })_{\lambda /<\omega}^{3}$,

then there is for each $n$ a free ideal $J_{n}$ and a cofinal family $\mathcal{A}_{n} \subset\left\langle J_{n}\right\rangle$ such that TWO does not have a winning n-tactic, but does have a winning $n+1$-tactic in $M G\left(\mathcal{A}_{n}, J_{n}\right)$.

Proof. Let $\lambda$ and $(L,<)$ be as in the hypotheses. It follows from Propositions 3 and 4 of 52 that there is for each integer $m>1$ a linearly ordered set $\left(L_{n},<_{n}\right)$ such that $\omega<\operatorname{cof}\left(L_{n},<_{n}\right)$ and:

$$
\left(L_{n},<_{n}\right) \rightarrow(\omega-\text { path })_{\lambda /<\omega}^{n}
$$

but

$$
\left(L_{n},<_{n}\right) \not \rightarrow(\omega-\text { path })_{\lambda /<\omega}^{n+1}
$$

Let $n>1$ and $\left(L_{n},<_{n}\right)$ be fixed for the rest of the proof. We may assume that the underlying set, $L_{n}$, is disjoint from $\mathcal{P}(\mathcal{P}(\lambda)) \cup \mathcal{P}(\lambda) \cup \lambda$.

Define a free ideal $J_{n}$ as follows: The underlying set on which $J_{n}$ lives, say $S_{n}$, is $[\lambda]^{<\aleph_{0}} \cup L_{n}$. For each $\alpha \in \lambda$ let $X_{\alpha}$ be the set $\left\{Z \in[\lambda]^{<\aleph_{0}}: \alpha \notin Z\right\}$. Let $\mathcal{T}$ be $\left\{X_{\alpha}: \alpha \in \lambda\right\}$. Put a subset $X$ of $S_{n}$ in $J_{n}$ if:

$X \cap[\lambda]^{<\aleph_{0}}$ is a subset of a union of finitely many elements

of $\mathcal{T}$, and $X \cap L_{n}$ is bounded above.

Then the cofinality of $\left\langle J_{n}\right\rangle$ is $\operatorname{cof}\left(L_{n},<_{n}\right)$. Define $\mathcal{A}_{n}$ so that $X \in$ $\mathcal{A}_{n}$ if:

$$
X \cap L_{n}=\left\{t \in L_{n}: t<z\right\} \text { for some } z \in L_{n} .
$$

Then $\mathcal{A}_{n}$ is cofinal in $\left\langle J_{n}\right\rangle$.

Claim 1: TWO does not have a winning $n$-tactic in $M G\left(\mathcal{A}_{n}, J_{n}\right)$. 
For let $\Phi$ be an $n$-tactic of TWO. For $x \in L_{n}$ put $V_{x}=[\lambda]^{<\aleph_{0}} \cup\{y \in$ $\left.L_{n}: y<_{n} x\right\}$. Define a partition $\Psi:\left[L_{n}\right]^{n} \rightarrow[\lambda]^{<\aleph_{0}}$ so that

$$
\left(\Phi\left(V_{x_{1}}\right) \cup \Phi\left(V_{x_{1}}, V_{x_{2}}\right) \cup \ldots \cup \Phi\left(V_{x_{1}}, \ldots, V_{x_{n}}\right)\right) \cap[\lambda]^{<\aleph_{0}}
$$

is a subset of $\cup\left\{X_{\alpha}: \alpha \in \Psi\left(\left\{x_{1}, \ldots, x_{n}\right\}\right)\right\}$.

By (1) we obtain an $\omega$-path $x_{1}<_{n} x_{2}<_{n} \ldots<_{n} x_{k}<_{n} \ldots$ and a finite set $F \subset \lambda$ such that $\Psi\left(x_{j+1}, \ldots, x_{j+n}\right) \subseteq F$ for all $j$. For each $m$ we define: $O_{m}=[\lambda]^{<\aleph_{0}} \cup V_{x_{m}}$. Letting $\left(O_{1}, T_{1}, \ldots, O_{k}, T_{k}, \ldots\right)$ be the corresponding $\Phi$-play, we find that TWO has lost this play since $[\lambda]^{<\aleph_{0}} \cap\left(\cup_{m=1}^{\infty} T_{m}\right) \subseteq \cup_{\alpha \in F} X_{\alpha} \neq[\lambda]^{<\aleph_{0}}$.

It follows that TWO does not have a winning $n$-tactic.

Claim 2: TWO has a winning $n+1$-tactic in $M G\left(\mathcal{A}_{n}, J_{n}\right)$.

First observe that $\cup_{\alpha \in F} X_{\alpha}=[\lambda]^{<\aleph_{0}}$ whenever $F$ is an infinite subset of $\lambda$.

Here is a definition of an $n+1$-tactic for TWO in this game: Let $\left\{t_{\alpha}: \alpha<\lambda\right\}$ enumerate $[\lambda]^{<\aleph_{0}}$ bijectively. Let $\Phi:\left[L_{n}\right]^{n+1} \rightarrow \lambda$ be a coloring which witnesses that $\left(L_{n},<_{n}\right) \not \rightarrow(\omega-\text { path })_{\lambda /<\omega}^{n+1}$. For each $X$ in $\mathcal{A}_{n}$ let $\phi_{X}$ be that element of $L_{n}$ for which $X \cap L_{n}=\left\{t \in L_{n}\right.$ : $\left.t<\phi_{X}\right\}$.

For $U_{1} \subset \ldots \subset U_{n+1}$ elements of $\mathcal{A}_{n}$, observe that $\phi_{U_{1}} \leq \ldots \leq$ $\phi_{U_{n+1}}$. For $X \subset Y$ sets in $\mathcal{A}_{n}$ such that $X \cap[\lambda]^{<\aleph_{0}} \neq Y \cap[\lambda]^{<\aleph_{0}}$ we set $\Psi(X, Y)=\min \left\{\alpha: t_{\alpha} \in Y \backslash X\right\}$.

Let $U_{1} \subset \ldots \subset U_{n+1} \in \mathcal{A}_{n}$ be given. We define:

1. $G\left(U_{1}, \ldots, U_{j}\right)=\emptyset$ when $j<n+1$,

2. $G\left(U_{1}, \ldots, U_{n+1}\right)=X_{\alpha} \cup\left(L_{n} \cap U_{n+1}\right)$ when $\phi_{U_{1}}<\ldots<\phi_{U_{n+1}}$, and $\Phi\left(\left\{\phi_{U_{1}}, \ldots, \phi_{U_{n+1}}\right\}\right)=\alpha$,

3. $G\left(U_{1}, \ldots, U_{n+1}\right)=X_{\alpha} \cup\left(L_{n} \cap U_{n+1}\right)$ where $\alpha$ is minimal such that $t_{\alpha} \in U_{i+1} \backslash U_{i}$ for some $i \leq n$, otherwise.

We show that $G$ is a winning $n+1$-tactic for TWO. Thus, let

$$
\left(O_{1}, T_{1}, \ldots, O_{m}, T_{m}, \ldots\right)
$$

be a $G$-play of the game. For typographical convenience we define:

1. $x_{i}=\phi_{O_{i}}$ for each $i$, and

2. $\alpha_{i}=\Psi\left(O_{i}, O_{i+1}\right)$ for each $i$ for which this is defined.

There are two cases to consider.

CASE 1: $\left\{i: x_{i}=x_{i+1}\right\}$ is finite.

Choose $m$ such that $x_{i}<x_{i+1}$ for all $i \geq m$. Then the set

$$
\left\{\Phi\left(\left\{x_{m+k+1}, \ldots, x_{m+k+n+1}\right\}\right): k=1,2, \ldots\right\}
$$

is an infinite subset of $\lambda$ and it follows from 2 . in the definition of $G$ that this play is won by TWO. 
CASE 2: $\left\{i: x_{i}=x_{i+1}\right\}$ is infinite.

Then the set $\left\{i: \Psi\left(O_{i}, O_{i+1}\right)\right.$ is defined $\}$ is infinite. But then it follows from 3 . in the definition of $G$ that TWO wins this play.

The hypotheses of Theorem 21 are realized under any of the following axiomatic circumstances (one uses Corollary 27 and Proposition 29 of S2] :

1. $2^{<\mathfrak{c}}=\mathfrak{c}+E H$,

2. $\mathfrak{c}<2^{\aleph_{1}}$, i.e., the negation of $L H$ (Lusin's second Continuum Hypothesis)

3. There is an infinite regular cardinal number $\kappa$ such that $2^{\kappa}=\kappa^{+}$.

For the case when $\lambda=\omega$, the example constructed in the proof of Theorem 21 shows that hypothesis 2 of Theorem 17 is to some extent necessary. This is because:

1. $\mathcal{A}_{\backslash}$ has the coherent decomposition property: For choose $\alpha_{1}<\alpha_{2}<$ $\ldots<\alpha_{n}<\ldots$ from $\omega$, and set $T_{m}=X_{\alpha_{1}} \cup \ldots \cup X_{\alpha_{m}}$ for each $m$. Then $[\omega]^{<\aleph_{0}}=\cup_{m=1}^{\infty} X_{\alpha_{m}}$, and $X_{\alpha_{j}} \subseteq X_{\alpha_{i}}$ for $j<i$. For $A \in \mathcal{A}_{\backslash}$ we put $A_{m}=\left(A \cap T_{m}\right) \cup\left(A \cap L_{n}\right)$.

2. $\left(\mathcal{A}_{m}, \subset\right) \rightarrow(\omega-\text { path })_{\omega /<\omega}^{m}$, but

3. $\left(\mathcal{A}_{m}, \subset\right) \not \rightarrow(\omega-\text { path })_{\omega /<\omega}^{m+1}$.

At this point it is an open problem whether the hypotheses (and for that matter the conclusion) of Theorem 21 are satisfied simply in the theory ZFC (see Problem 9 of $\mathrm{S} 2$ ).

\subsection{The game $\mathrm{MG}(\mathrm{J})$}

$M G(J)$ denotes the version of $M G(\mathcal{A}, J)$ where $\langle J\rangle=\mathcal{A}$. In Problem 1 of [S1] it was asked whether there is for each $k$ a free ideal $J_{k}$ such that TWO does not have a winning $k$-tactic in $M G\left(J_{k}\right)$, but does have a winning $k+1$-tactic in $M G\left(J_{k}\right)$. This problem is still open. In [S1], Corollary 10, it was proven that TWO does not have a winning 2-tactic in the game $M G\left(J_{\mathbb{R}}\right)$, but that TWO has a winning 3-tactic in $M G\left(J_{\mathbb{R}}\right)$ if for example the Continuum Hypothesis is assumed. We now extend these results in two directions.

1. In Problem 3 of that paper it was asked if player TWO has a winning 3 -tactic if instead of the Continuum Hypothesis one uses the theory $Z F C+M A+E H+\neg C H$, which is explained below. We now show that the answer is affirmative.

2. We identify circumstances under which TWO does not have a winning $k$-tactic in $M G\left(J_{\mathbb{R}}\right)$ for any $k$; combining this with a consistency result of Todorcevic (given in the appendix), it follows that it is also consistent that there is no $k$ for which TWO has a winning $k$-tactic in $M G\left(J_{\mathbb{R}}\right)$. 
It follows that the existence of a winning $k$-tactic for TWO in $M G\left(J_{\mathbb{R}}\right)$ is not decided by the axioms of traditional set theory. One might now wonder if it is consistent that for example TWO does not have a winning 3-tactic in $M G\left(J_{\mathbb{R}}\right)$, but does have a winning 4-tactic? This is not possible since a theorem of $\mathrm{S} 3$ implies that either TWO has a winning 3-tactic, or else there is no $k$ such that TWO has a winning $k$-tactic in $M G\left(J_{\mathbb{R}}\right)$.

Let $E H$ (which abbreviates Embedding Hypothesis) denote the statement:

every linearly ordered set of cardinality $\leq \mathfrak{c}$ embeds in $\left({ }^{\omega} \omega, \ll\right)$.

The hypothesis $E H$ is a consequence of the Continuum Hypothesis. Laver has proven ( $\mathrm{L}$ ) that the theory $Z F C+E H+\neg C H$ is consistent, and Woodin (W], pp. 31-47), extending this, has proven the consistency of the theory $Z F C+M A+E H+\neg C H$. This theory implies that $2^{<\mathfrak{c}}=\mathfrak{c}+E H$, which in turn is strong enough to prove that the partition relation

$$
(\mathcal{P}(\mathfrak{c}), \subset) \nrightarrow \rightarrow(\omega-\text { path })_{\omega /<\omega}^{3}
$$

holds (see S2], top of p. 60). Thus we have:

Proposition 22 The theory " $Z F C+\neg C H+T W O$ has a winning 3-tactic in $M G\left(J_{\mathbb{R}}\right)$ " is consistent.

Proof. Consider any model of $Z F C+E H+\neg C H+2^{<\mathfrak{c}}=\mathfrak{c}$ in which $\left\langle J_{\mathbb{R}}\right\rangle$ has a cofinal chain. Let $\mathcal{C}$ denote this cofinal chain. By Theorem 14 we may assume that this cofinal chain has a coherent decomposition and that it satisfies the partition relation $(\mathcal{C}, \subset) \not \rightarrow(\omega-\text { path })_{\omega /<\omega}^{2}$. Since we also have $(\mathcal{P}(\mathfrak{c}), \subset) \nrightarrow \nrightarrow(\omega-\text { path })_{\omega /<\omega}^{3}$ it follows that:

1. $\left(\left\langle J_{\mathbb{R}}\right\rangle, \subset\right) \nrightarrow \rightarrow(\omega-\text { path })_{\omega /<\omega}^{3}$, and

2. The family $\left\langle J_{\mathbb{R}}\right\rangle$ has a coherent decomposition.

Theorem 17 implies that TWO has a winning 3 -tactic in $M G\left(\left\langle J_{\mathbb{R}}\right\rangle, J_{\mathbb{R}}\right)$. This completes the proof of the proposition.

Indeed, our proof of Proposition shows more generally that if $J$ is a free ideal on a set of cardinality at most $\mathfrak{c}$, and if $\langle J\rangle$ has a cofinal chain and the coherent decomposition property, then the theory $Z F C+E H+2^{<\mathfrak{c}}=\mathfrak{c}$ proves that TWO has a winning 3-tactic in $M G(J)$. This generalizes Theorem $8(\mathrm{a})$ of $\mathrm{S} 1$.

Next we give hypotheses under which there is no $k$ for which TWO has a winning $k$-tactic in $M G\left(J_{\mathbb{R}}\right)$. In the appendix we give a proof that these hypotheses are consistent with $Z F C$. This consistency result is due to Todorcevic.

Theorem 23 Assume that $\operatorname{cof}\left(J_{\mathbb{R}}, \subset\right)=\lambda$ and that the partition relation $(\mathcal{P}(\mathfrak{c}), \subset) \rightarrow(\omega-\text { path })_{\lambda /<\omega}^{3}$ holds. Then there is no $k$ for which TWO has a winning $k$-tactic in $M G\left(J_{\mathbb{R}}\right)$. 
Proof. Let $k$ as well as a $k$-tactic $F$ for $T W O$ be given. Let $X$ be a nowhere dense subset of cardinality $\mathfrak{c}$ of $\mathbb{R} \backslash \mathbb{Q}$. Let $\mathcal{A}=\left\{A_{\alpha}: \alpha<\lambda\right\}$ be a bijectively enumerated cofinal subfamily of $J_{\mathbb{R}}$.

Define a partition $\Phi:[\mathcal{P}(X)]^{k} \rightarrow \lambda$ so that

$$
\Phi\left(\left\{X_{1}, \cdots, X_{k}\right\}\right)=\beta
$$

where $\beta$ is minimal such that

$$
F\left(\mathbb{Q} \cup X_{1}\right) \cup \cdots \cup F\left(\mathbb{Q} \cup X_{1}, \cdots, \mathbb{Q} \cup X_{k}\right) \subset A_{\beta} .
$$

Since $(\mathcal{P}(\mathfrak{c}), \subset) \rightarrow(\omega-\text { path })_{\lambda /<\omega}^{3}$, it follows that $(\mathcal{P}(\mathfrak{c}), \subset) \rightarrow(\omega-$ path $)_{\lambda /<\omega}^{k}$ ( see [S2], Proposition 36). Accordingly, choose a finite set $G \subset \lambda$ and an increasing $\omega$-sequence $X_{1} \subset X_{2} \subset \cdots$ of subsets of $X$ such that $\Phi\left(\left\{X_{j+1}, \cdots, X_{j+k}\right\}\right) \in G$ for all $j$. Put $O_{n}=X_{n} \cup \mathbb{Q}$ for all $n$. Let $B$ be the nowhere dense set $\cup\left\{A_{\alpha}: \alpha \in G\right\}$. Also define $T_{j}=F\left(O_{1} \cdots, O_{j}\right)$ for $j \leq k$, and $T_{j+k}=F\left(O_{j+1}, \cdots, O_{j+k}\right)$ for all $j$. Then

$$
\left(O_{1}, T_{1}, O_{2}, T_{2}, \cdots\right)
$$

is an $F$-play of $M G\left(J_{\mathbb{R}}\right)$ for which $\mathbb{Q} \subset \cup_{n=1}^{\infty} O_{n}$ and $\cup_{n=1}^{\infty} T_{n} \subseteq B$. Since $B$ is nowhere dense, $\mathbb{Q} \backslash B \neq \emptyset$. It follows that TWO has lost this play.

We now consider games of the form $M G\left([\kappa]^{<\lambda}\right)$. In Proposition 15 of S1] it was shown that if TWO has a winning $k$-tactic in this game for some $k$, then TWO in fact has a winning 3-tactic. It is not known if " 3 " is optimal (this is Problem 7 of [S1]). It also follows from [S1], Proposition 5, that if $\lambda \rightarrow(\omega-\text { path })_{\omega /<\omega}^{2}$, then TWO does not have a winning $k$-tactic in this game for any $k$. We now present slightly sharper results.

Theorem 24 Let $\lambda$ be an uncountable cardinal number of countable cofinality. Let $k>1$ be an integer. The following statements are equivalent:

1. Player TWO has a winning k-tactic in the game $M G\left(\left[\lambda^{+}\right]^{<\lambda}\right)$.

2. $\left(\left[\lambda^{+}\right] \leq \lambda, \subset\right) \not \rightarrow(\omega-\text { path })_{\omega /<\omega}^{k}$.

3. $\lambda^{+} \nrightarrow \rightarrow(\omega-\text { path })_{\omega /<\omega}^{2}$ and $(\mathcal{P}(\lambda), \subset) \not \rightarrow(\omega-\text { path })_{\omega /<\omega}^{k}$.

Proof. By Theorem 1 and Proposition 15 of [S1] we may assume that $k \in\{2,3\}$. Let $\lambda_{1}<\ldots<\lambda_{n}<\ldots$ be a sequence of cardinal numbers converging to $\lambda$.

1. $\Rightarrow 2$.

Let $F$ be a winning $k$-tactic for TWO in $M G\left(\left[\lambda^{+}\right]^{<\lambda}\right)$. Put $S=$ $\lambda^{+} \backslash \lambda$. Define a coloring $\Phi:\left[[S]^{\leq \lambda}\right]^{k} \rightarrow \omega$ so that

$$
\Phi\left(X_{1}, \ldots, X_{k}\right)=\min \left\{n: \mid F\left(\lambda \cup X_{1}, \ldots, \lambda \cup X_{k} \mid \leq \lambda_{n}\right\} .\right.
$$

Since $F$ is a winning $k$-tactic for TWO, $\Phi$ is a coloring which witnesses the partition relation in 2 . 
2. $\Rightarrow 1$.

According to Corollary 16, $\left(\left[\lambda^{+}\right]^{<\lambda}, \subset\right)$ has the coherent decomposition property. Since $\left[\lambda^{+}\right]^{\leq \lambda}$ has a cofinal chain it follows that this family of sets itself has a coherent decomposition. The partition property in 2 implies that the family $\left[\lambda^{+}\right]^{\leq \lambda}$ satisfies the hypotheses of Theorem 17; thus TWO has a winning $k$-tactic in $M G\left(\left[\lambda^{+}\right]^{<\lambda}\right)$.

The equivalence of 2 . and 3 . is also easy to establish.

Corollary 25 Let $\lambda$ be an uncountable cardinal number of countable cofinality. Assume $Z F C+E H+\lambda<\mathfrak{c}+\mathfrak{c}=2^{<\mathfrak{c}}$. Then TWO has a winning 2-tactic in $M G\left(\left[\lambda^{+}\right]^{<\lambda}\right)$.

Proof. The hypothesis $E H+\mathfrak{c}=2^{<\mathfrak{c}}$ implies that both $\lambda^{+}$and $(\mathcal{P}(\lambda), \subset)$ embed in $\left({ }^{\omega} \omega, \ll\right)$ for any $\lambda<\mathfrak{c}$. It then follows from Corollary 13 of S2 that the partition relations in 3. of Theorem 24 hold for $k=2$ for each $\lambda<\mathfrak{c}$.

\subsection{The game $\mathrm{SMG}(\mathrm{J})$}

For a free ideal $J$ on an infinite set $S$, the game $S M G(J)$ (read "strongly monotonic game on J") is defined so that an $\omega$-sequence $\left(O_{1}, T_{1}, \ldots, O_{n}, T_{n}, \ldots\right)$ is a play if for each $n$,

1. $O_{n} \in\langle J\rangle$ is player ONE's move in inning $n$,

2. $T_{n} \in J$ is player TWO's move in inning $n$, and

3. $O_{n} \cup T_{n} \subseteq O_{n+1}$.

Player TWO wins this play if $\cup_{n=1}^{\infty} O_{n}=\cup_{n=1}^{\infty} T_{n}$.

Throughout this section we assume that $\langle J\rangle$ is a proper ideal on $S$.

Theorem 26 Let $J \subset \mathcal{P}(S)$ be a free ideal and let $\mathcal{A}$ be a cofinal subfamily of $\langle J\rangle$ such that:

1. TWO has a winning $k$-tactic in $M G(\mathcal{A}, J)$,

2. there are functions $\Phi_{1}:\langle J\rangle \rightarrow J$ and $\Phi_{2}:\langle J\rangle \rightarrow \mathcal{A}$ such that:

(a) $A \subset \Phi_{2}(A)$ for each $A \in\langle J\rangle$, and

(b) $\Phi_{2}(A) \subset \Phi_{2}(B)$ whenever $A \cup \Phi_{1}(A) \subseteq B \in\langle J\rangle$.

Then TWO has a winning 2-tactic in $S M G(J)$.

Proof Let $\mathcal{A}, \Phi_{1}$ and $\Phi_{2}$ be as in the hypotheses. For each $A$ in $\langle J\rangle$ define $\left(A_{1}, \ldots, A_{k}\right)$ so that $A_{1}=\Phi_{2}(A)$ and $A_{j+1}=\Phi_{2}\left(A_{j}\right)$ for each $j<k$. Also define: $\Psi(A)=\Phi_{1}(A) \cup \Phi_{1}\left(A_{1}\right) \cup \ldots \cup \Phi_{1}\left(A_{k}\right)$.

Let $F$ be a winning $k$-tactic for TWO in $M G(\mathcal{A}, J)$. Define a $k$ tactic, $G$, for TWO as follows. Let $A \subset B$ be given. 
CASE 1: $G(A)=F\left(A_{1}\right) \cup \ldots \cup F\left(A_{1}, \ldots, A_{k}\right) \cup \Psi(A)$.

CASE 2: If $A_{k} \subset B_{1}$, we let $G(A, B)$ be the set

$F\left(A_{2}, \ldots, A_{k}, B_{1}\right) \cup F\left(A_{3}, \ldots, A_{k}, B_{1}, B_{2}\right) \cup \ldots \cup F\left(B_{1}, \ldots, B_{k}\right) \cup \Psi_{1}(B)$.

CASE 3: Otherwise we put $G(A, B)=G(B)$.

Then $G$ is a winning 2-tactic for TWO in $S M G(J)$. For let

$$
\left(O_{1}, T_{1}, \ldots, O_{n}, T_{n}, \ldots\right)
$$

be a play of $S M G(J)$ during which TWO followed the 2-tactic $G$. For each $j$ we put $M_{j}^{1}=\Phi_{2}\left(O_{j}\right), \ldots, M_{j}^{k}=\Phi_{2}\left(M_{j}^{k-1}\right)$. An inductive computation shows that

- $\left(M_{1}^{1}, M_{1}^{2}, \ldots, M_{1}^{k}, M_{2}^{1}, M_{2}^{2}, \ldots, M_{2}^{k}, \ldots\right)$ is a sequence of legal moves for $O N E$ in the game $M G(\mathcal{A}, J)$, and that

- 1. $F\left(M_{1}^{1}\right) \cup \ldots \cup F\left(M_{1}^{1}, \ldots, M_{1}^{k}\right) \subseteq T_{1}$, and

2. $F\left(M_{j}^{1}, \ldots, M_{j}^{k}\right) \cup F\left(M_{j}^{2}, \ldots, M_{j}^{k}, M_{j+1}^{1}\right) \cup \ldots \cup F\left(M_{j}^{k}, M_{j+1}^{1}, \ldots, M_{j+1}^{k-1}\right)$ $\subseteq T_{j+1}$ for each $j$.

Since $F$ is a winning $k$-tactic for TWO in the game $M G(\mathcal{A}, J)$, and since $\cup_{n=1}^{\infty} O_{n} \subseteq \cup_{n=1}^{\infty} M_{n}^{1}$, TWO won the given play of $S M G(J)$.

The next corollary solves Problems 10 and 11 of [S1].

Corollary 27 Player $T W O$ has a winning 2-tactic in the game $S M G\left(J_{\mathbb{R}}\right)$.

Proof. Fix, by Corollary 18, a cofinal family $\mathcal{A} \subset\left\langle J_{\mathbb{R}}\right\rangle$ such that TWO has a winning 2-tactic in $M G\left(\mathcal{A}, J_{\mathbb{R}}\right)$.

We define $\Phi_{1}:\left\langle J_{\mathbb{R}}\right\rangle \rightarrow J_{\mathbb{R}}$ and $\Phi_{2}:\left\langle J_{\mathbb{R}}\right\rangle \rightarrow \mathcal{A}$ as follows:

Fix $X \in\left\langle J_{\mathbb{R}}\right\rangle$, and choose a sequence $\left(X_{0}, X_{1}, \ldots, X_{n}, \ldots\right)$ such that:

1. $X_{0}=X$,

2. $X_{n+1} \in \mathcal{A}$ and $\mathbb{N} \cdot X_{n} \subseteq X_{n+1}$

for each $n$. Put $\Phi_{2}(X)=\cup_{n=1}^{\infty} X_{n}$.

Fix $X \in\left\langle J_{\mathbb{R}}\right\rangle$ and let $\Phi_{1}(X)$ be a nowhere dense set for which $\Phi_{2}(X) \subset \mathbb{N} \cdot \Phi_{1}(X)$.

Then $\mathcal{A}, \Phi_{1}$ and $\Phi_{2}$ are as required by Theorem 26 .

Corollary 28 For each of the ideals $J_{n}$ constructed in the proof of Theorem 21, TWO has a winning 2-tactic in $S M G\left(J_{n}\right)$.

Proof. Let $\mathcal{A}_{n}$ be as in the proof of Theorem 21. For each $X \in\left\langle J_{n}\right\rangle$ we let $\Phi_{2}(X)$ be an element of $\mathcal{A}_{n}$ which contains it, and we let $\Phi_{1}(X)=\left\{a_{X}\right\}$ where $a_{X} \in L_{n} \backslash \Phi_{2}(X)$. Then $\mathcal{A}_{n}, \Phi_{1}$ and $\Phi_{2}$ are as required by Theorem 26 . 
Before giving another application of Theorem 26 we give an example of free ideals $J$ which show that TWO does not always have a winning $k$ tactic in the game $S M G(J)$ for some $k$. These examples are also relevant to the material of the next section. The symbol $M(\omega, 2)$ denotes the smallest ordinal $\alpha$ for which the partition relation $\alpha \rightarrow(\omega-\text { path })_{\omega /<\omega}^{2}$ holds. $M(\omega, 2)$ is a regular cardinal less than or equal to $\mathfrak{c}^{+}$. It in fact satisfies the partition relation $M(\omega, 2) \rightarrow(\omega-\text { path })_{\omega /<\omega}^{n}$ for all $n$. Let $\kappa$ be an initial ordinal number. It is consistent that $M(\omega, 2)$ is equal to $\aleph_{2}$ while $\mathfrak{c}$ is larger than $\kappa$ (this is yet another result of Todorcevic).

Theorem 29 Let $\lambda$ be a cardinal number of countable cofinality and let $\kappa$ be a cardinal number larger than $\lambda$. If $M(\omega, 2) \leq \lambda^{+}$, then there is no $k$ such that player TWO has a winning $k$-tactic in $S M G\left([\kappa]^{<\lambda}\right)$.

Proof. Let $F$ be a $k$-tactic for TWO.

Player ONE's counter-strategy will be to play judiciously chosen subsets from $\kappa$. We first single out those sets from which ONE will make moves.

Choose sets $S_{0} \subset S_{1} \subset \ldots \subset S_{\alpha} \subset \ldots \in[\kappa]^{\lambda}$ for $\alpha<\lambda^{+}$such that:

1. $\lambda \subset S_{0}$,

2. $\cup\left\{F\left(S_{i_{1}}, \ldots, S_{i_{j}}\right): j \leq k, i_{1}<\ldots<i_{j}<\alpha\right\} \subset S_{\alpha}$ for each $0<\alpha<\lambda^{+}$.

Now let $\lambda_{1}<\lambda_{2}<\ldots<\lambda$ be an increasing sequence of regular cardinal numbers converging to $\lambda$. Define a function $\Gamma:\left[\lambda^{+}\right]^{k} \rightarrow \omega$ so that

$$
\Gamma\left(\xi_{1}, \ldots, \xi_{k}\right)=\min \left\{m:\left|F\left(S_{\xi_{1}}, \ldots, S_{\xi_{k}}\right)\right| \leq \lambda_{m}\right\}
$$

Then, on account of the relation $M(\omega, 2) \leq \lambda^{+}$, choose an $m<$ $\omega$ and a sequence $\alpha_{k+1}<\ldots<\alpha_{k+m}<\ldots$ from $\lambda^{+}$such that $\Gamma\left(\alpha_{j+1}, \ldots, \alpha_{j+k}\right) \leq m$ for all $j$.

Consider the sequence

$$
\left(S_{\alpha_{1}}, F\left(S_{\alpha_{1}}\right), \ldots, S_{\alpha_{k}}, F\left(S_{\alpha_{1}}, \ldots, S_{\alpha_{k}}\right), \ldots, S_{\alpha_{k+m}}, F\left(S_{\alpha_{1+m}}, \ldots, S_{\alpha_{k+m}}\right), \ldots\right) .
$$

It is a play of the game $S M G\left([\kappa]^{<\lambda}\right)$ during which TWO used the $k$ tactic $F$. To see that TWO lost this play, let $T_{k}$ denote TWO's $k$-th move. The choice of the sequence $\alpha_{k+1}<\ldots$ implies that $\cup_{n=1}^{\infty} T_{n}$ has cardinality less than $\lambda$. The union of the sets played by ONE has cardinality $\lambda$; TWO didn't catch up with ONE.

Corollary 30 For $\omega=\operatorname{cof}(\lambda) \leq \lambda<\kappa$ cardinal numbers with $\operatorname{cof}\left([\kappa]^{\leq \lambda}, \subset\right.$ )$=\kappa$, the following statements are equivalent:

1. TWO has a winning 2-tactic in $S M G\left([\kappa]^{<\lambda}\right)$.

2. $\lambda^{+} \nrightarrow \rightarrow(\omega-\text { path })_{\omega /<\omega}^{2}$. 
Proof. It follows from Theorem 29 that 1 . implies 2.

That 2. implies 1.:

By the cofinality hypothesis and by 2 . we find, according to Corollary 20, a well-founded cofinal family $\mathcal{A}$ such that TWO has a winning 2 -tactic in $M G\left(\mathcal{A},[\kappa]^{<\lambda}\right)$. We may assume that there is an enumeration $\left\{A_{\alpha}: \alpha<\kappa\right\}$ of $\mathcal{A}$ for which $\alpha \in A_{\alpha}$ for each $\alpha$. Define $\Phi_{1}$ and $\Phi_{2}$ as follows:

For $X \in[\kappa]^{\leq \lambda}$ define a sequence $\left(X_{0}, \ldots, X_{m}, \ldots\right)$ such that:

1. $X_{0}=X$, and

2. $X_{n+1}=\cup_{\alpha \in X_{n}} A_{\alpha}$

for each $n$.

Choose $\Phi_{2}(X) \in \mathcal{A}$ such that $\cup_{n<\omega} X_{n} \subseteq \Phi_{2}(X)$.

Pick $z_{X} \in\left(\kappa \backslash \Phi_{2}(X)\right)$ and pick $\rho_{X}$ minimal such that $\rho_{X} \notin \Phi_{2}(X)$, and $\Phi_{2}(X) \subset A_{\rho_{X}}$. Put $\Phi_{1}(X)=\left\{z_{X}, \rho_{X}\right\}$.

Then $\mathcal{A}, \Phi_{1}$ and $\Phi_{2}$ are as required by Theorem 26 .

This result will be discussed at greater length after Theorem 34

We finally mention that it is still unknown whether there is for each $m$ a free ideal $J_{m}$ such that TWO does not have a winning $m$-tactic, but does have a winning $m+1$-tactic in $S M G\left(J_{m}\right)$. This is Problem 9 of S1. In this connection it is worth noting the following relationship between winning $k$-tactics in $M G(J)$ and winning $m$-tactics in $S M G(J)$. The proof uses ideas as in the proof of Theorem 26.

Theorem 31 If TWO has a winning k-tactic in $M G(J)$, then TWO has a winning 2-tactic in $S M G(J)$.

\subsection{The game $\operatorname{VSG}(\mathrm{J})$}

For a free ideal $J$ on an infinite set $S$, the game $V S G(J)$ (read "very strong game on J") is defined so that an $\omega$-sequence $\left(O_{1},\left(T_{1}, S_{1}\right), \ldots, O_{n},\left(T_{n}, S_{n}\right), \ldots\right)$ is a play if for each $n$,

1. $O_{n} \in\langle J\rangle$ is player ONE's move in inning $n$,

2. $\left(T_{n}, S_{n}\right) \in J \times\langle J\rangle$ is player TWO's move in inning $n$, and

3. $O_{n} \cup T_{n} \cup S_{n} \subseteq O_{n+1}$.

Player TWO wins this play if $\cup_{n=1}^{\infty} O_{n}=\cup_{n=1}^{\infty} T_{n}$.

We assume for this section that $\langle J\rangle$ is also a proper ideal on $S$. Given a cofinal family $\mathcal{A} \subset\langle J\rangle$, we may assume whenever convenient that ONE is playing from $\mathcal{A}$ in the game $V S G(J)$. It is clear that if TWO has a winning $k$-tactic in $S M G(J)$, then TWO has a winning $k$-tactic is $V S G(J)$. The converse is not so clear. 
Problem 1 Let $J$ be a free ideal on a set $S$ and let $k$ be a positive integer. Is it true that if TWO has a winning $k$-tactic in $V S G(J)$, then TWO has a winning $k$-tactic in $S M G(J)$ ?

In the next theorem we find a partial converse.

Theorem 32 Let $J$ be a free ideal on a set $S$ and let $k$ be a positive integer. If $\operatorname{add}(\langle J\rangle, \subset)=\operatorname{cof}(\langle J\rangle, \subset)$, then the following statements are equivalent:

1. TWO has a winning 2-tactic in $S M G(J)$.

2. TWO has a winning $k$-tactic in $S M G(J)$.

3. TWO has a winning $k$-tactic in $V S G(J)$.

Proof That 1. and 2. are equivalent: This is Theorem 19 of $\mathrm{S1}$.

That 2. implies 3.: Let $F$ be a winning $k$-tactic for TWO in $S M G(J)$. Define $G$ so that

$$
G\left(A_{1}, \ldots, A_{j}\right)=\left(F\left(A_{1}, \ldots, A_{j}\right), A_{j} \cup F\left(A_{1}, \ldots, A_{j}\right)\right)
$$

for $j \leq k$. Then $G$ is a winning $k$-tactic for TWO in $V S G(J)$.

That 3. implies 2.: Let $G$ be a winning $k$-tactic for TWO in $V S G(J)$. Then choose a sequence $\left(M_{\xi}: \xi<\operatorname{cof}(\langle J\rangle, \subset)\right)$ such that:

1. $M_{\xi} \subset M_{\nu}$ for $\xi<\nu<\operatorname{cof}(\langle J\rangle, \subset)$ and

2. $\left\{M_{\xi}: \xi<\operatorname{cof}(\langle J\rangle, \subset)\right\}$ is cofinal in $\langle J\rangle$.

Now $\operatorname{cof}(\langle J\rangle, \subset)$ is a regular uncountable cardinal number. We may thus further assume that the sequence $\left(M_{\xi}: \xi<\operatorname{cof}(\langle J\rangle, \subset)\right)$ has been chosen such that if $(U, T)=G\left(M_{\xi_{1}}, \ldots, M_{\xi_{j}}\right)$, then $U \cup T \subset M_{\eta}$ for all $\xi_{j}<\eta<\operatorname{cof}(\langle J\rangle, \subset)$.

For each $X \in\langle J\rangle$ define $\alpha(X)=\min \left\{\xi: X \subset M_{\xi}\right\}$. For each $\xi$ choose $z_{\xi} \in S \backslash M_{\xi}$. We now define a $k$-tactic, $F$, for TWO in $S M G(J)$.

Let $X_{1} \subset \ldots \subset X_{j} \in\langle J\rangle$ for a $j \leq k$ be given.

CASE 1: $\alpha\left(X_{1}\right)<\ldots<\alpha\left(X_{j}\right)$. Let $(U, T)=G\left(M_{\alpha\left(X_{1}\right)}, \ldots, M_{\alpha\left(X_{j}\right)}\right)$ and define $F\left(X_{1}, \ldots, X_{j}\right)=U \cup\left\{z_{\alpha\left(X_{j}\right)+1}\right\}$.

CASE 2: Otherwise, set $F\left(X_{1}, \ldots, X_{j}\right)=\left\{z_{\alpha\left(X_{j}\right)+1}\right\}$. Then $F$ is a winning $k$-tactic for TWO in $S M G(J)$.

There is the following analogue of Theorem 26 for the very strong game:

Proposition 33 Let $J$ be a free ideal on a set $S$. If there is a cofinal family $\mathcal{A} \subset\langle J\rangle$ such that $T W O$ has a winning $k$-tactic in $M G(\mathcal{A}, J)$, then TWO has a winning 2-tactic in $V S G(J)$.

Proof. Let $\mathcal{A} \subset\langle J\rangle$ be a cofinal family such that TWO has a winning $k$-tactic in $M G(\mathcal{A}, J)$. We will define a winning 2-tactic for TWO for the game $V S G(J)$. To this end, choose a winning $k$-tactic, $F$, for TWO for the game $M G(\mathcal{A}, J)$. For each $X \in\langle J\rangle$ choose a set 
$A_{1}(X) \subset \ldots \subset A_{k}(X)$ from $\mathcal{A}$ such that $X \subset A_{1}(X)$, and choose $\Psi(X)$ from $\mathcal{A}$ such that $A_{k}(X) \subset \Psi(X)$.

Let $X \subset Y$ be sets from $\langle J\rangle$.

CASE 1: $G(X)=\left(F\left(A_{1}(X)\right) \cup \ldots \cup F\left(A_{1}(X), \ldots, A_{k}(X)\right), \Psi(X)\right)$.

CASE 2: Define $G(X, Y)$ so that:

1. $G(X, Y)=\left(F\left(A_{2}(X), \ldots, A_{k}(X), A_{1}(Y) \cup \ldots \cup F\left(A_{1}(Y), \ldots, A_{k}(Y)\right), \Psi(Y)\right)\right.$

if $\Psi(X) \subset Y$, and

2. $G(X, Y)=G(Y)$ otherwise.

Then $G$ is a winning 2-tactic for TWO in $V S G(J)$. For let $\left(O_{1},\left(T_{1}, S_{1}\right), O_{2},\left(T_{2}, S_{2}\right), \ldots\right)$ be a play of $V S G(J)$ such that $\left(T_{1}, S_{1}\right)=G\left(O_{1}\right)$ and $\left(T_{n+1}, S_{n+1}\right)=$ $G\left(O_{n}, O_{n+1}\right)$ for all $n$. Then $S_{n}=\Psi\left(O_{n}\right)$ and $A_{k}\left(O_{n}\right) \subset A_{1}\left(O_{n+1}\right)$ for each $n$. An inductive computation, using this information, shows that TWO won this play of $V S G(J)$.

Combining Theorem 32 and Theorem 29 we see that TWO does not always have a winning $k$-tactic in games of the form $V S G(J)$. Combining Theorem 32 and Corollary 30 we obtain another game-theoretic characterization of the partition relation $\lambda^{+} \rightarrow(\omega-\text { path })_{\omega /<\omega}^{2}$ when $\lambda$ is an uncountable cardinal of countable cofinality.

Analogous to the case of the ideal of countable subsets of an infinite set, there is for each uncountable cardinal number $\lambda$ which is of countable cofinality, a proper class of cardinals $\kappa$ for which the ideal $[\kappa] \leq \lambda$ has the irredundancy property. It is also a consequence of $M A+\mathfrak{c}>\lambda$ that the partition relation $\lambda^{+} \not \rightarrow(\omega-\text { path })_{\omega /<\omega}^{2}$ holds. Accordingly it is consistent that there is a proper class of cardinals $\kappa$ such that TWO has a winning 2-tactic in the game $\operatorname{VSG}\left([\kappa]^{\leq \lambda}\right.$ ). The following problem (to be compared with the upcoming Conjecture 1) is open.

Problem 2 Let $\lambda$ be an uncountable cardinal of countable cofinality. Is it true that if TWO has a winning 2-tactic in the game $V S G\left(\left[\lambda^{+}\right]^{<\lambda}\right)$, then TWO has a winning 2-tactic in $V S G\left([\kappa]^{<\lambda}\right)$ for all $\kappa>\lambda$ ?

Our next theorem (Theorem 34) applies to abstract free ideals whose $\sigma$-completions have small principal bursting number. It is not clear to us whether " 3 " occurring in Theorem 34 is optimal. One of its applications is that $\mathrm{ZFC}+\mathrm{GCH}$ implies that TWO has a winning 3-tactic in $V S G\left([\kappa]^{<\aleph_{0}}\right)$ for all $\kappa$. It is very likely that the " 3 " appearing in this application is not optimal, as will be discussed later.

Theorem 34 Let $J$ be a free ideal such that:

1. $b u(\langle J\rangle, \subset)=\aleph_{2}$,

2. $\operatorname{add}(\langle J\rangle, \subset)=\aleph_{1}$,

3. $\operatorname{cof}(\langle J\rangle, \subset)=\lambda$ and

4. $[\lambda]^{<\aleph_{0}}$ has the coherent decomposition property.

Then player TWO has a winning 3-tactic in $V S G(J)$. 
Proof. Let $J$ be a free ideal (on a set $S$ ) as in the hypotheses. Let $\mathcal{A}$ be a well-founded cofinal family of cardinality $\lambda$, such that $\mid\{B \in \mathcal{A}$ : $B \subseteq A\} \mid \leq \aleph_{1}$ for each $A \in \mathcal{A}$.

For each $A \in \mathcal{A}$ fix $\nu_{A} \leq \omega_{1}$ and a bijective enumeration $\left\{J_{\xi}(A)\right.$ : $\left.\xi<\nu_{A}\right\}$ of the set $\{X \in \mathcal{A}: X \subseteq A\}$.

Choose a sequence $\left(C_{\xi}: \xi<\omega_{1}\right)$ from $\langle J\rangle$ such that:

1. $C_{\xi} \subset C_{\nu}$ for $\xi<\nu$ and

2. $\cup_{\xi<\omega_{1}} C_{\xi} \notin\langle J\rangle$.

For $A \in \mathcal{A}$ define $\xi_{A}=\min \left\{\xi<\omega_{1}: C_{\xi} \not \subset A\right\}$.

For $A \subset B$ elements from $\mathcal{A}$, define a set $\tau(A, B)$ such that $\left(S_{1}, \ldots, S_{n}\right)$ is in $\tau(A, B)$ if:

1. $2 \leq n<\omega$,

2. $S_{1}=B$ and $S_{2}=A$,

3. $S_{j+1} \in\left\{J_{\xi}\left(S_{j}\right): \xi<\nu_{S_{j}}\right.$ and $\left.C_{\xi} \subset S_{j-1}\right\}$ for $2 \leq j<n$.

For $\left(S_{1}, \ldots, S_{n}\right)$ and $\left(T_{1}, \ldots, T_{m}\right)$ in $\tau(A, B)$ define $\left(S_{1}, \ldots, S_{n}\right)<$ $\left(T_{1}, \ldots, T_{m}\right)$ if $n<m$ and $\left(S_{1}, \ldots, S_{n}\right)=\left(T_{1}, \ldots, T_{n}\right)$. Then $(\tau(A, B),<$ ) is a tree. Each branch of this tree is finite since $(\mathcal{A}, \subset)$ is wellfounded. Indeed, $\tau(A, B)$ is a countable set.

Define $F(A, B)$ to be the set of $X \in \mathcal{A}$ such that $X \in\left\{S_{1}, \ldots, S_{m}\right\}$ for some $\left(S_{1}, \ldots, S_{m}\right) \in \tau(A, B)$. Then $F(A, B)$ is a countable set. Notice that if $C \subset A \subset B$ are elements of $\mathcal{A}$ such that $C \in\left\{J_{\xi}(A)\right.$ : $\xi \leq \nu_{A}$ and $\left.C_{\xi} \subset B\right\}$, then $F(C, A) \subset F(A, B)$.

Let $\mathcal{B} \subset[\mathcal{A}]^{\aleph_{0}}$ be cofinal, well-founded and with the coherent decomposition property. For each $B \in \mathcal{B}$ choose a decomposition $B=\cup_{n=1}^{\infty} B^{n}$ where each $B^{n}$ is finite, and these decompositions satisfy the coherent decomposition requirement. By Proposition 15 of 52] we also fix a function

$$
\mathcal{K}:[\mathcal{B}]^{2} \rightarrow \omega
$$

which witnesses that $(\mathcal{B}, \subset) \nrightarrow \rightarrow(\omega-\text { path })_{\omega /<\omega}^{2}$.

Define $\Phi_{1}:[\mathcal{A}]^{2} \rightarrow \mathcal{B}$ such that

$\left(\cup\left\{F(X, Y):\left(\exists\left(S_{1}, \ldots, S_{n}\right) \in \tau(A, B)\right)\left(X \subset Y\right.\right.\right.$ and $\left.\left.\left.X, Y \in\left\{S_{1}, \ldots, S_{n}\right\}\right)\right\}\right)$

is a subset of $\Phi_{1}(A, B)$. Also define $\Phi_{2}:[\mathcal{A}]^{2} \rightarrow \mathcal{A}$ such that

$$
C_{\xi} \cup C_{\xi_{B}} \cup\left(\cup \Phi_{1}(A, B)\right) \subset \Phi_{2}(A, B)
$$

where now $A=J_{\xi}(B)$.

Note that if $A, B$, and $C$ are elements of $\mathcal{A}$ such that $A \subset B \subset$ $\Phi_{2}(A, B) \subset C$, then $\Phi_{1}(A, B) \subset \Phi_{1}(B, C)$.

Finally, choose for each $A \in \mathcal{A}$ a $\Phi_{3}(A) \in \mathcal{A}$ such that $A \cup C_{\xi_{A}} \subseteq$ $\Phi_{3}(A)$. 
Choose for each $A \in \mathcal{A}$ a sequence of sets $A^{0} \subseteq \ldots, A^{n} \subseteq \ldots$ such that each $A^{i}$ is in $J$ and $A=\cup_{n=0}^{\infty} A^{n}$.

We now define a 3-tactic for TWO: First note that for the very strong game we may make the harmless assumption that player ONE's moves are all from the cofinal family $\mathcal{A}$. Let $A \subset B \subset C$ be sets from $\mathcal{A}$. Here are player TWO's responses $\mathcal{F}(A), \mathcal{F}(A, B)$ and $\mathcal{F}(A, B, C)$ :

Case 1: $\mathcal{F}(A)=\left(\emptyset, \Phi_{3}(A)\right)$

Case 2: $\mathcal{F}(A, B)=\left(\emptyset, \Phi_{2}(A, B)\right)$

Case 3: $\mathcal{F}(A, B, C)=\left(D, \Phi_{2}(B, C)\right)$

if $\Phi_{2}(A, B) \subseteq C$, where $D=C_{1}^{m} \cup \ldots \cup C_{r}^{m}$ is given by: $m \geq$ $\mathcal{K}\left(\left\{\Phi_{1}(A, B), \Phi_{1}(B, C)\right\}\right)$ is minimal such that $\left(\Phi_{1}(A, B)\right)^{n} \subseteq\left(\Phi_{1}(B, C)\right)^{n}$ for all $n \geq m$, and $\left(\Phi_{1}(B, C)\right)^{m}=\left\{C_{1}, \ldots, C_{r}\right\}$.

Case 4: In all other cases define $\mathcal{F}(A, B, C)=\mathcal{F}(B, C)$.

To see that $\mathcal{F}$ is a winning 3 -tactic for TWO, consider a play

$$
\left(O_{1},\left(T_{1}, S_{1}\right), O_{2},\left(T_{2}, S_{2}\right), \ldots\right)
$$

of $\operatorname{VSG}(J)$ for which

1. $\left(T_{1}, S_{1}\right)=\mathcal{F}\left(O_{1}\right)$,

2. $\left(T_{2}, S_{2}\right)=\mathcal{F}\left(O_{1}, O_{2}\right)$ and

3. $\left(T_{n+3}, S_{n+3}\right)=\mathcal{F}\left(O_{n+1}, O_{n+2}, O_{n+3}\right)$

for all $n$.

Then $T_{1}=T_{2}=\emptyset, S_{1}=\Phi_{3}\left(O_{1}\right), S_{2}=\Phi_{2}\left(O_{1}, O_{2}\right)$ and $S_{n+1}=$ $\Phi_{2}\left(O_{n}, O_{n+1}\right)$ for all $n \geq 2$. From the fact that $O_{n} \supseteq S_{n-1}$ for all $n \geq 2$ it follows that

$$
O_{1} \subset O_{2} \subset \Phi_{2}\left(O_{1}, O_{2}\right) \subseteq O_{3} \subset \Phi_{2}\left(O_{2}, O_{3}\right) \subseteq O_{4} \subset \ldots,
$$

whence $\Phi_{1}\left(O_{1}, O_{2}\right) \subset \Phi_{1}\left(O_{2}, O_{3}\right) \subset \Phi_{1}\left(O_{3}, O_{4}\right) \subset \ldots$ For each $k$ let $m_{k}$ denote the minimal integer such that

1. $\mathcal{K}\left(\left\{\Phi_{1}\left(O_{k}, O_{k+1}\right), \Phi_{1}\left(O_{k+1}, O_{k+2}\right)\right\}\right) \leq m_{k}$ and

2. $\left(\Phi_{1}\left(O_{k}, O_{k+1}\right)\right)^{n} \subseteq\left(\Phi\left(O_{k+1}, O_{k+2}\right)\right)^{n}$ for all $n \geq m_{k}$.

From the properties of $\mathcal{K}$ it follows that there are infinitely many $k$ such that $m_{j}<m_{k}$ for each $j<k$. Fix $i$, and fix the smallest $j \geq i$ such that $O_{i} \in \Phi_{1}\left(O_{j}, O_{j+1}\right)$. Then let $t$ be minimal such that $O_{i} \in\left(\Phi_{1}\left(O_{j}, O_{j+1}\right)\right)^{t}$. Then for each $k$ such that $m_{\ell}<m_{k}$ for each $\ell<k$, and $t<m_{k}, O_{i}^{m_{k}} \subseteq T_{k}$. It follows that $O_{i} \subseteq \cup_{n=1}^{\infty} T_{n}$. From this it follows that TWO won this $\mathcal{F}$-play of $\operatorname{VSG}(J)$. 
Corollary 35 (GCH) For every infinite cardinal number $\kappa$, TWO has a winning 3 -tactic in $\operatorname{VSG}\left([\kappa]^{<\aleph_{0}}\right)$.

The results of Corollaries 30 and 35 should be compared with those of Koszmider [Ko for the game $M G\left([\kappa]^{<\aleph_{0}}\right)$. In Corollary 30 we show that there is a proper class of $\kappa$ such that TWO has a winning 2-tactic in $S M G\left([\kappa]^{<\aleph_{0}}\right)$, and thus in $V S G\left([\kappa]^{<\aleph_{0}}\right)$. This class includes $\aleph_{n}$ for all $n<\omega$. In Ko it is proven that TWO has a winning 2-tactic in $M G\left(\left[\aleph_{n}\right]^{<\aleph_{0}}\right)$ for all $n \in \omega(\sqrt[K R]{\mathrm{Ko}}$, Theorem 18). Under the additional set theoretic assumption that both $\lambda$ holds and $\lambda^{\aleph_{0}}=\lambda^{+}$for all uncountable cardinal numbers $\lambda$ which are of countable cofinality, Koszmider further proves that player TWO has a winning 2-tactic in $M G\left([\kappa]^{<\aleph_{0}}\right)$ for all $\kappa$ ([Ko, Theorem 19). In light of these results it is consistent that TWO has a winning 2-tactic in the game $S M G\left([\kappa]^{<\aleph_{0}}\right)$ and thus in the game $V S G\left([\kappa]^{<\aleph_{0}}\right)$ for all $\kappa$.

All this evidence leads us to believe that one could prove (without recourse to additional set theoretic hypotheses) that player TWO has a winning 2-tactic in the game $V S G\left([\kappa]^{<\aleph_{0}}\right)$ for all infinite $\kappa$. We suspect even more: that TWO has a winning 2-tactic in $S M G\left([\kappa]^{<\aleph_{0}}\right)$ for all $\kappa$. We state this formally as a conjecture:

Conjecture 1 Player TWO has a winning 2-tactic in the game $S M G\left([\kappa]^{<\aleph_{0}}\right)$ for each infinite cardinal number $\kappa$.

One can modify the proof of Theorem 34 to obtain the following result:

Theorem 36 Let $J$ be a free ideal on a set $S$ such that

1. $b u(\langle J\rangle, \subset)=\aleph_{n}$ for some finite $n$,

2. there is an $\left(\omega_{k}, \omega_{k}\right)$-pseudo Lusin set in $(\langle J\rangle, \subset)$ for each $k \in\{1, \ldots, n\}$,

3. $\operatorname{cof}(\langle J\rangle, \subset)=\lambda)$, and

4. $\left([\lambda]^{<\aleph_{0}}, \subset\right)$ has the coherent decomposition property.

Then player TWO has a winning $n+1$-tactic in $V S G(J)$.

We now give an example which shows, assuming the Continuum Hypothesis, that the hypothesis that $\operatorname{add}(\langle J\rangle, \subset)=\aleph_{1}$ of Theorem 34 is necessary (see Corollary 38).

Theorem 37 Let $\omega_{\alpha}$ be the initial ordinal corresponding with $\mathfrak{c}$. Then there is a free ideal $J \subset \mathcal{P}\left(\omega_{\alpha+1}\right)$ such that $\operatorname{cof}(\langle J\rangle, \subset)=\aleph_{\alpha+1}$ and there is no positive integer $k$ for which TWO has a winning $k$-tactic in $V S G(J)$.

Proof Define $J \subset \mathcal{P}\left(\omega_{\alpha+1}\right)$ such that $X \in J$ if, and only if, $|X| \leq \aleph_{\alpha}$ and $X \cap \omega$ is finite. Then $\operatorname{cof}(\langle J\rangle, \subset)=\operatorname{add}(\langle J\rangle, \subset)=\omega_{\alpha+1}$. By Theorem 32 it suffices to show that TWO doesn't have a winning 2-tactic in $S M G(J)$.

Let $F$ be a 2-tactic for TWO in $S M G(J)$. For $\omega<\eta<\omega_{\alpha+1}$ put $\phi(\eta)=\sup (\eta \cup F(\eta))$. Let $C \subseteq \omega_{\alpha+1} \backslash(\omega+1)$ be a closed unbounded set such that $\phi(\gamma)<\beta$ whenever $\gamma<\beta$ are in $C$. 
For each $\eta \in C$ define $\phi_{\eta}: C \backslash(\eta+1) \rightarrow \omega_{\alpha+1}$ so that $\phi_{\eta}(\beta)=$ $\sup (\beta \cup F(\eta, \beta))$ for all $\beta$. Then choose a closed, unbounded set $C_{\eta} \subseteq C \backslash(\alpha+1)$ such that $\phi_{\eta}(\beta)<\gamma$ whenever $\beta<\gamma$ are in $C_{\eta}$.

Let $D$ be the diagonal intersection of $\left(C_{\eta}: \eta \in C\right)$; i.e., $D=\{\xi \in$ $C: \xi \in \cap\left\{C_{\eta}: \eta<\xi\right.$ and $\left.\eta \in C\right\}$. Then $D$ is an unbounded subset of $\omega_{\alpha+1}$. Now observe that if $\eta_{1}<\eta_{2}<\eta_{3}$ are elements of $D$, then

1. $\eta_{2} \in C_{\eta_{1}}$,

2. $\eta_{3} \in C_{\eta_{1}} \cap C_{\eta_{2}}$, and thus

3. $F\left(\eta_{1}\right) \subseteq \eta_{2}$ and $F\left(\eta_{1}, \eta_{2}\right) \subseteq \eta_{3}$.

Define $\Phi:[D]^{2} \rightarrow \omega$ so that

$$
\Phi(\eta, \beta)=\max (\omega \cap(F(\eta) \cup F(\eta, \beta))) .
$$

By the Erdös-Rado theorem we obtain an $n<\omega$ and an uncountable $X \subset D$ such that $\Phi(\eta, \beta)=n$ for all $\eta<\beta \in X$. Pick $\eta_{1}<\eta_{2}<$ $\ldots<\eta_{m}<\ldots$ from $X$ and put $O_{n}=\eta_{n}$ for each $n$. Put $T_{1}=F\left(O_{1}\right)$ and $T_{n+1}=F\left(O_{n}, O_{n+1}\right)$ for each $n$.

Then $\left(O_{1}, T_{1}, \ldots, O_{n}, T_{n}, \ldots\right)$ is an $F$-play of $S M G(J)$ which is lost by TWO.

Corollary 38 Assume the Continuum Hypothesis. Then there is a free ideal $J \subset \mathcal{P}\left(\omega_{2}\right)$ such that $\operatorname{cof}(\langle J\rangle, \subset)=\aleph_{2}$, and there is no positive integer $k$ for which TWO has a winning $k$-tactic in $V S G(J)$.

We don't know if there is for each $m$ a free ideal $J_{m}$ such that TWO does not have a winning $m$-tactic, but does have a winning $m+1$-tactic in $\operatorname{VSG}\left(J_{m}\right)$.

Problem 3 Is there for each $m$ a free ideal $J_{m}$ such that TWO does not have a winning $m$-tactic, but does have a winning $m+1$-tactic in $\operatorname{VSG}\left(J_{m}\right)$ ?

\subsection{The Banach-Mazur game and an example of Debs}

The Banach-Mazur game is defined as follows for a topological space $(X, \tau)$. Players ONE and TWO alternately choose nonempty open subsets from $X$; in the n-th inning player ONE first chooses $O_{n}$ and TWO responds with $T_{n}$. An inning is played for each positive integer. The sets chosen by the players must satisfy the rule

$$
O_{n+1} \subseteq T_{n} \subseteq O_{n}
$$

for all n. Player TWO wins the play

$$
\left(O_{1}, T_{1}, \ldots, O_{n}, T_{n}, \ldots\right)
$$

if the intersection of these sets is nonempty; otherwise player ONE wins. Following Galvin and Telgarsky G-T, we denote this game by $B M(X, \tau)$. 
In the early 1980's Debs [D] solved Problem 3 of $\mathrm{F}-\mathrm{K}$ by giving examples of topological spaces $(X, \tau)$ for which player TWO has a winning strategy in the game $B M(X, \tau)$, but no winning 1-tactic. In all but one of Debs' examples it was known (in $Z F C$ ) that TWO has a winning 2-tactic. We show here that also for the remaining example player TWO has a winning 2 -tactic (Corollary 43). This was previously known under the assumption of some additional hypotheses.

This result eliminates this example as a candidate for providing evidence (consistent, modulo $Z F C$ ) towards the following conjecture of Telgarsky:

Conjecture 2 (Telgarsky, $[\mathbf{T}]$, p. 236) For each positive integer $k$ there is a topological space $\left(X_{k}, \tau_{k}\right)$ such that TWO does not have a winning $k$ tactic, but does have a winning $k+1$-tactic in the game $B M\left(X_{k}, \tau_{k}\right)$.

The following unpublished result of Galvin is the only theorem known to us which gives general conditions under which TWO has a winning 2-tactic if TWO has a winning strategy in the Banach-Mazur game:

Theorem 39 (Galvin, unpublished) Let $(X, \tau)$ be a topological space for which TWO has a winning strategy in the Banach-Mazur game. If this space has a pseudo base $\mathcal{P}$ with the property that

- $|\{V \in \mathcal{P}: B \subseteq V\}|<s(B)$ for each $B$ in $\mathcal{P}$,

then TWO has a winning 2-tactic.

Here the cardinal number $s(B)$ is defined to be the minimal $\kappa$ such that $B$ does not contain a collection of $\kappa$ pairwise disjoint nonempty open subsets; it is said to be the Souslin number of $B$.

This subsection is organised as follows. We first prove a theorem concerning $k$-tactics in the Banach-Mazur game which is analogous to Theorem 5 of G-T. It provides an equivalent formulation of Telgarsky's conjecture which allows player TWO slightly more information: TWO may also remember the inning number. After this we give our result on Debs' example.

\subsubsection{Markov $k$-tactics.}

Whereas a $k$-tactic for player TWO remembers at most the latest $k$ moves of the opponent, a strategy for TWO which remembers in addition to this information also the number of the inning in progress is called a Markov $k$-tactic. This choice of terminology is by analogy with the terminology "tactic" (used by Choquet [C], p. 116, Definition 7.11 for what we call a 1-tactic) and "Markov strategy" (used by Galvin and Telgarsky [G-T], p. 52 for what we call a Markov 1-tactic). A k-tactic is the special case of a Markov k-tactic where the inning number is ignored by the player.

Note that if $(X, \tau)$ has a dense set of isolated points then player TWO has a winning 1-tactic in $B M(X, \tau)$. Thus we may assume that if at all possible, player ONE will avoid playing an open set which contains an isolated point. From the point of view of $k$-tactics for TWO we may therefore 
restrict our attention to topological spaces without isolated points. By the following proposition we may further restrict our attention to topological spaces in which each nonempty open set contains infinitely many pairwise disjoint open subsets.

Proposition 40 Let $(X, \tau)$ be a topological space with no infinite set of pairwise disjoint open subsets. Then there is a positive integer $n$ such that:

$$
\tau \backslash\{\emptyset\}=\tau_{1} \cup \ldots \cup \tau_{n}
$$

where each $\tau_{i}$ has the finite intersection property.

Proof. Claim 1: There is a positive integer $\mathrm{n}$ such that every collection of pairwise disjoint nonempty open subsets is of cardinality $\leq n$.

Proof of Claim 1: This is a well known fact: see e.g. [C-N, Lemma 2.10, p. 31 .

Now let $n$ be the minimal positive integer satisfying Claim 1. Let $\mathcal{U}=\left\{U_{1}, \ldots, U_{n}\right\}$ be a collection of pairwise disjoint nonempty open subsets of the space. Then $\mathcal{U}$ is a maximal pairwise disjoint family. For $1 \leq i \leq n$, let $\tau_{i}$ be a maximal family of nonempty open sets such that:

1. $U_{i} \in \tau_{i}$,

2. any two elements of $\tau_{i}$ have nonempty intersection.

Claim 2: $\tau \backslash\{\emptyset\}=\tau_{1} \cup \ldots \cup \tau_{n}$

Proof of Claim 2: Assume the contrary and let $Y$ be a nonempty open set which is in none of the $\tau_{i}$. Then we find for each $i$ an $X_{i}$ in $\tau_{i}$ which is disjoint from $Y$ (by maximality of each $\tau_{i}$ ). We may assume that $X_{i} \subseteq U_{i}$ for each $i$. But then $\left\{X_{1}, \ldots, X_{n}, Y\right\}$ is a collection of $n+1$ pairwise disjoint nonempty open subsets of $(X, \tau)$, contradicting the choice of $n$.

Each $\tau_{i}$ has the finite intersection property.

Proposition 41 Let $(X, \tau)$ be a topological space for which:

1. Player $T W O$ has a winning strategy in the game $B M(X, \tau)$ and

2. every collection of pairwise disjoint open subsets is finite.

Then TWO has a winning 1-tactic in $B M(X, \tau)$.

Proof. Write, by Proposition 40

$$
\tau \backslash\{\emptyset\}=\tau_{1} \cup \ldots \cup \tau_{n}
$$

where each $\tau_{i}$ has the finite intersection property, and $n$ is minimal. Choose a pairwise disjoint collection $\left\{U_{1}, \ldots, U_{n}\right\}$ such that $U_{j} \in \tau_{j}$ for each $j$.

Claim 3: For each $j$, if $S_{1} \supseteq S_{2} \supseteq \ldots$ is a denumerable chain from $\tau_{j}$, then $\cap_{n=1}^{\infty} S_{n} \neq \emptyset$. 
Proof of Claim 3: Assume the contrary, and fix $j$ and a chain $S_{1} \supseteq$ $S_{2} \supseteq \ldots$ in $\tau_{j}$ such that $\cap_{n=1}^{\infty} S_{n}=\emptyset$. We may assume that $S_{n+1} \subset$ $S_{n} \subset U_{j}$ for all $n$.

Let $F$ be a winning perfect information strategy for TWO in $B M(X, \tau)$. Consider the play

$$
\left(O_{1}, T_{1}, \ldots, O_{m}, T_{m}, \ldots\right)
$$

which is defined so that:

1. $O_{1}=S_{1}$,

2. $T_{m}=F\left(O_{1}, \ldots, O_{m}\right)$ for all $m$ and

3. $O_{m+1}=T_{m} \cap S_{m+1}$.

Note that since each $S_{m}$ is a subset of $U_{j}$, each response by player TWO using $F$ is a member of $\tau_{j}$, whence each $O_{m}$ is a legal move by ONE. We now get the contradiction that TWO lost this play despite the fact that TWO was playing according to a winning strategy. This completes the proof of Claim 3.

We now define a winning 1-tactic, $G$, for TWO. Let $U$ be a nonempty open subset of $X$. Choose the minimal $j$ such that $U_{j} \cap U \neq \emptyset$ and put $G(U)=U_{j} \cap U$. Claim 3 implies that this is a winning 1-tactic for TWO.

Theorem 42 Let $k$ be a positive integer. If player TWO has a winning Markov $k$-tactic in the Banach Mazur game on some topological space, then TWO has a winning k-tactic in the Banach-Mazur game on that space.

Proof. Let $k$ be a positive integer and let $(X, \tau)$ be a topological space such that TWO has a winning Markov $k$-tactic in the game $B M(X, \tau)$. We may assume that this space has no isolated points. By Proposition 41 we may also assume that every nonempty open subset of $X$ contains infinitely many pairwise disjoint open subsets (player ONE may safely avoid playing open subsets not having this property). By Theorem 5 of G-T we may assume that $k>1$.

Let $F$ be a winning Markov $k$-tactic for TWO. For each nonempty open set $U$, let $\left\{J_{m}(U): 0<m<\omega\right\}$ bijectively enumerate a collection of infinitely many pairwise disjoint nonempty open subsets of $U$.

Define a $k$-tactic $G$ for TWO as follows. Let $U_{1} \supseteq \ldots \supseteq U_{j}$ be nonempty open sets, where $1 \leq j \leq k$.

Case 1: $j=1$ : Put $G\left(U_{1}\right)=F\left(J_{2}\left(U_{1}\right), 1\right)$.

Case 2: $j>1$ and $U_{i+1} \subseteq J_{l+i+1}\left(U_{i}\right)$ for $1 \leq i<j$, for some $l$. Put $G\left(U_{1}, \ldots, U_{j}\right)=F\left(J_{l+2}\left(U_{1}\right), \ldots, J_{l+j+1}\left(U_{j}\right), l+j\right)$.

Case 3: In all other cases define $G\left(U_{1}, \ldots, U_{j}\right)=G\left(U_{j}\right)$. 
To see that $G$ is a winning $k$-tactic for TWO, consider a play

$$
\left(O_{1}, T_{1}, \ldots, O_{m}, T_{m}, \ldots\right)
$$

such that

- $T_{j}=G\left(O_{1}, \ldots, O_{j}\right)$ for $j \leq k$ and

- $T_{n+k}=G\left(O_{n+1}, \ldots, O_{n+k}\right)$ for all $n$.

From the definition of $G$ and the rules of the Banach-Mazur game it follows that $T_{1}$ is defined by Case 1 and $T_{m}$ for $m>1$ by Case 2 . In particular, writing $S_{n}$ for $J_{n+1}\left(O_{n}\right)$ we find that:

1. $T_{j}=F\left(S_{1}, \ldots, S_{j}, j\right)$ for $j \leq k$ and

2. $T_{n+k}=F\left(S_{n+1}, \ldots, S_{n+k}, n+k\right)$

for all n. Indeed,

$$
O_{1} \supseteq S_{1} \supseteq T_{1} \supseteq O_{2} \supseteq S_{2} \supseteq \ldots
$$

Since $F$ is a winning Markov $k$-tactic, it follows that $\cap_{n=1}^{\infty} O_{n} \neq \emptyset$.

\subsubsection{Debs' example}

Let $\sigma$ be the topology of the real line whose elements are of the form $U \backslash M$ where $U$ is open and $M$ is meager in the usual topology. The symbol $B M(\mathbb{R}, \sigma)$ denotes the Banach-Mazur game, played on the topological space $(\mathbb{R}, \sigma)$. It is known that TWO has a winning strategy but does not have a winning 1-tactic in $B M(\mathbb{R}, \sigma)$.

Corollary 43 Player TWO has a winning 2-tactic in the game $B M(\mathbb{R}, \sigma)$.

Proof. Theorem 22 of [31] and Corollary 27.

\section{Appendix: Consistency of the hypothe- ses of Theorem [23].}

We start with a ground model $V$ and let $\mathbf{P} \in V$ be a forcing notion of cardinality $\leq \mathfrak{c}$. For a cardinal $\kappa$, denote by $\mathbf{P}_{\kappa}$ the product of $\kappa$ copies of $\mathbf{P}$ taken side-by-side with countable supports.

Lemma 44 Let $\lambda$ be an uncountable cardinal. Suppose:

1. $\kappa \geq \kappa_{1} \geq \kappa_{2} \geq \kappa_{3} \geq \omega_{2}$ are cardinal numbers such that

- $\kappa$ is a regular cardinal,

- $\kappa \rightarrow\left(\kappa_{1}\right)_{\lambda}^{2}$,

- $\kappa_{1} \rightarrow\left(\kappa_{2}\right)_{\mathfrak{c}}^{3}$,

- $\kappa_{2} \rightarrow\left(\kappa_{3}\right)_{\lambda}^{2}$ and 
- $\kappa_{3} \rightarrow\left(\omega_{2}\right)_{\mathfrak{c}}^{3}$.

2. Forcing with $\mathbf{P}$ adds a real to the ground model.

Then $\mathfrak{c} \rightarrow(\omega-\text { path })_{\lambda /<\omega}^{2}$ holds in the forcing extension $V^{\mathbf{P}_{\kappa}}$.

Proof. Let $\lambda, \kappa, \kappa_{1}, \ldots, \kappa_{3}, \mathbf{P}$ be as in the assumptions. Our argument closely follows section 2 of [To1].

For sets $A, B$ the symbol $A / B$ denotes $\{\{\alpha, \beta\}: \alpha \in A, \beta \in B, \alpha<$ $\beta\}$.

Note that $V^{\mathbf{P}_{\kappa}}$ satisfies $\mathfrak{c}=\kappa$; we prove that $\kappa \rightarrow(\omega-\text { path })_{\lambda /<\omega}^{2}$ holds in $V^{\mathbf{P}_{\kappa}}$.

Let $[\kappa]^{2}=\bigcup_{i<\lambda} \dot{K}_{i}$ be a given partition in $V^{\mathbf{P}_{\kappa}}$. Let $\dot{U}$ be a $\mathbf{P}_{\kappa^{-}}$ name for a member of $[\kappa]^{\kappa}$. Pick $A \in[\kappa]^{\kappa}$ and for each $\alpha \in A$, a $q_{\alpha} \in \mathbf{P}_{\kappa}$ such that $q_{\alpha} \|-\alpha \in \dot{U}$ and such that the $q_{\alpha}$ 's form a $\Delta$-system. Define $H:[A]^{2} \rightarrow(\lambda+1)$ so that $H(\{\alpha, \beta\})=i$ if $i$ is the minimal $j$ such that $p \|-\{\alpha, \beta\} \in \dot{K}_{j}$ for some $p \leq q_{\alpha}, q_{\beta}$ if such $j$ exists (i.e., if $q_{\alpha}$ and $q_{\beta}$ are compatible), and $H(\{\alpha, \beta\})=\lambda$ if $q_{\alpha}$ is incompatible with $q_{\beta}$.

By our choice of $\kappa$, the partition relation $\kappa \rightarrow\left(\kappa_{1}\right)_{\lambda}^{2}$ holds. Therefore, choose $A_{1} \subset[A]^{\kappa_{1}}$ and $i \leq \lambda$ such that $H^{\prime \prime}\left[A_{1}\right]^{2}=\{i\}$. Since $\mathbf{P}_{\kappa}$ satisfies the $\mathfrak{c}^{+}$-c.c., we have $i<\lambda$.

Let $\left\langle p_{\alpha, \beta}:\{\alpha, \beta\} \in\left[A_{1}\right]^{2}\right\rangle$ be a fixed sequence of conditions such that $p_{\alpha, \beta} \leq q_{\alpha}, q_{\beta}$ and $p_{\alpha, \beta} \|-\{\alpha, \beta\} \in \dot{K}_{i}$. For $\alpha<\beta<\gamma$ in $A_{1}$ we define $H_{0}(\{\alpha, \beta, \gamma\})$ to be a pair $(c, d)$, where $c \operatorname{codes} p_{\alpha, \beta}$ and $p_{\alpha, \gamma}$ as structures as well as relations between the ordinals of $\operatorname{dom}\left(p_{\alpha, \beta}\right)$ and $\operatorname{dom}\left(p_{\alpha, \gamma}\right)$, and $d$ does the same for $p_{\alpha, \gamma}$ and $p_{\beta, \gamma}$. Since there are only $\mathfrak{c}$ such pairs, and since $\kappa_{1} \rightarrow\left(\kappa_{2}\right)_{\mathfrak{c}}^{3}$ holds, choose $A_{2} \in\left[A_{1}\right]^{\kappa_{2}}$ and $(c, d)$ such that $H_{0}^{\prime \prime}\left[A_{2}\right]^{3}=\{(c, d)\}$. For convenience, assume that $A_{2}$ has order type $\kappa_{2}$. It follows that for each $\alpha \in A_{2}$ the sequence $\left\langle p_{\alpha, \beta}: \beta \in A_{2} \backslash(\alpha+1)\right\rangle$ forms a $\Delta$-system with root $p_{\alpha}^{0}$ $\left(\leq q_{\alpha}\right)$, and that for each $\gamma \in A_{2}$ the sequence $\left\langle p_{\beta, \gamma}: \beta \in A_{2} \cap \gamma\right\rangle$ forms a $\Delta$-system with root $p_{\gamma}^{1}\left(\leq q_{\gamma}\right)$. Moreover, the $p_{\alpha}^{0}$ 's and $p_{\gamma}^{1}$ 's form $\Delta$-systems with roots $p^{0}$ and $p^{1}$ respectively. To see the latter, note that we may shrink $A_{2}$ to a cofinal subset $A_{3}$ so that the relevant $p_{\alpha}^{0}$ 's and $p_{\alpha}^{1}$ 's do in fact form a $\Delta$-system. Now consider $\alpha, \beta, \gamma \in$ $A_{3}$, and $\alpha^{\prime}, \beta^{\prime} \in A_{2}$. Comparing $H_{0}(\{\alpha, \beta, \gamma\}), H_{0}\left(\left\{\alpha, \beta^{\prime}, \gamma\right\}\right)$ and $H_{0}\left(\left\{\alpha^{\prime}, \beta^{\prime}, \gamma\right\}\right)$, one sees that the sequence $\left\langle p_{\alpha}^{0}: \alpha \in A_{2}\right\rangle$ forms a $\Delta$-system. A similar argument works for the $p_{\gamma}^{1}$ 's.

Also, $p^{0}$ is compatible with $p^{1}$. We call $\left\langle p_{\alpha, \beta}:\{\alpha, \beta\} \in B / B\right\rangle$ a double $\Delta$-system with root $p^{0} \cup p^{1}$.

There is no reason why for a given $\alpha$ the conditions $p_{\alpha}^{0}$ and $p_{\alpha}^{1}$ should be compatible: if these were always compatible, our argument would yield a consistency proof of $\mathfrak{c} \rightarrow(\omega)_{\lambda}^{2}$, which is false in $Z F C$.

We now save as much of the compatibility between $p_{\alpha}^{0}$ and $p_{\alpha}^{1}$ as is needed for the consistency proof of $\mathfrak{c} \rightarrow(\omega-\text { path })_{\lambda /<\omega}^{2}$. Thin out $A_{2}$ to a cofinal subset $A_{3}$ such that $\operatorname{dom}\left(p_{\alpha}^{0} \cup p_{\alpha}^{1}\right) \cap \operatorname{dom}\left(p_{\beta}^{0} \cup p_{\beta}^{1}\right)=$ 
$\operatorname{dom}\left(p^{0} \cup p^{1}\right)$ for all $\{\alpha, \beta\} \in A_{3} / A_{3}$. Then in particular $p_{\alpha}^{1}$ and $p_{\beta}^{0}$ are compatible for $\{\alpha, \beta\} \in A_{3} / A_{3}$.

Now repeat the reasoning above with $A_{2}$ in place of $A, \kappa_{2}$ in place of $\kappa, \kappa_{3}$ in place of $\kappa_{1}$, and $\omega_{2}$ in place of $\kappa_{2}$. Also, $p_{\alpha}^{1}$ will now play the role of $q_{\alpha}$, and $p_{\beta}^{0}$ the role of $q_{\beta}$ for $\{\alpha, \beta\} \in A_{3} / A_{3}$. We get a set $A_{4} \subset A_{3}$ of order type $\omega_{2}$ and some $j<\lambda$ (which may be different from $i$ ), conditions $\bar{p}_{\alpha, \beta}$ for $\{\alpha, \beta\} \in A_{4} / A_{4}$ that form a double $\Delta$-system with root $\bar{p}^{0} \cup \bar{p}^{1}$, and we get roots $\bar{p}_{\alpha}^{0}$ and $\bar{p}_{\gamma}^{1}$ as before. Now $\bar{p}_{\alpha, \beta} \|-\{\alpha, \beta\} \in \dot{K}_{j}$ for $\{\alpha, \beta\} \in A_{4} / A_{4}$.

Our choice of $\bar{p}_{\alpha, \beta}$ at the beginning of the second run of the argument insures that $\bar{p}_{\alpha}^{0} \leq p_{\alpha}^{1}$ and $\bar{p}_{\gamma}^{1} \leq p_{\gamma}^{0}$, and hence $\bar{p}^{0} \leq p^{1}$ and $\bar{p}^{1} \leq p^{0}$.

Now let $\mathbf{G}$ be a generic subset of $\mathbf{P}_{\kappa}$. Define:

$\dot{X}=\left\{\alpha \in A_{4}: p_{\alpha}^{0} \in \mathbf{G}\right\}$,

$\dot{Y}=\left\{\alpha \in A_{4}: p_{\alpha}^{1} \in \mathbf{G}\right\}$,

$\dot{W}=\left\{\alpha \in A_{4}: \bar{p}_{\alpha}^{0} \in \mathbf{G}\right\}$,

$\dot{Z}=\left\{\alpha \in A_{4}: \bar{p}_{\alpha}^{1} \in \mathbf{G}\right\}$.

Then $\dot{Z} \subset \dot{X}$ and $\dot{W} \subset \dot{Y}$, and all four sets are cofinal in $A_{4}$.

Now $\bar{p}^{0} \cup \bar{p}^{1}$ forces the following facts:

(1) $\exists \delta_{1} \in \omega_{2} \forall \alpha \in \dot{X} \backslash \delta_{1}\left\{\beta \in \dot{W}:\{\alpha, \beta\} \in \dot{K}_{i}\right\}$ is cofinal in $A_{4}$, and

(2) $\exists \delta_{2} \in \omega_{2} \forall \alpha \in \dot{Y} \backslash \delta_{2}\left\{\beta \in \dot{Z}:\{\alpha, \beta\} \in \dot{K}_{j}\right\}$ is cofinal in $A_{4}$.

The combination of (1) and (2) suffices to construct in $V^{\mathbf{P}_{\kappa}}$ an $\omega$ path of the given partition that uses only colors $i$ and $j$ :

Let $\delta=\max \left\{\delta_{1}, \delta_{2}\right\}$. Inductively define an increasing sequence $\left\langle x_{n}\right.$ : $n \in \omega\rangle$ of ordinals such that $x_{2 k} \in Z$ (and hence in $X$ ), $x_{2 k+1} \in W$, and $\left\{x_{2 k}, x_{2 k+1}\right\} \in \dot{K}_{i}$ (by (1)); $\left\{x_{2 k+1}, x_{2 k+2}\right\} \in \dot{K}_{j}$ (by (2)).

It remains to prove (1) and (2). We shall prove (1) only; the proof of (2) is similar, and is a special case of [To1, section 2, property (1)].

Assume that $\bar{p}^{0} \cup \bar{p}^{1}$ does not force (1). Then we can find a condition $\bar{p}^{2} \leq \bar{p}^{0} \cup \bar{p}^{1}$ and a $\mathbf{P}_{\kappa}$-name $\dot{D} \in[\dot{X}]^{\omega_{2}}$ and for each $\beta \in \dot{D}$ a $\gamma_{\beta} \in A_{4} \backslash(\beta+1)$ such that $\bar{p}^{2} \|-\{\beta, \delta\} \notin \dot{K}_{i}$ whenever $\delta \in \dot{W} \backslash \gamma_{\beta}$.

Working in $V$, we pick $B \in\left[A_{4}\right]^{\omega_{2}}$ such that for each $\beta \in B$ we find $r_{\beta} \leq p_{\beta}^{0} \cup \bar{p}^{2}$ such that $r_{\beta} \|-\beta \in \dot{D}$, and $r_{\beta}$ decides the value of $\gamma_{\beta}$. We may assume that the $r_{\beta}$ 's form a $\Delta$-system with root $\leq \bar{p}^{2} \leq \bar{p}^{0} \cup \bar{p}^{1}$, and that $\gamma_{\beta}<\delta$ for all $\{\beta, \delta\} \in B / B$. Since $\left\langle p_{\alpha, \beta}: \beta \in B \backslash(\alpha+1)\right\rangle$ forms a $\Delta$-system, we may also assume that $\operatorname{dom}\left(r_{\beta}\right) \cap \operatorname{dom}\left(p_{\beta, \delta} \backslash p_{\beta}^{0}\right)=\emptyset$ for all $\delta>\gamma_{\beta}$ in $A_{4}$.

Pick $\delta \in A_{2}$ such that $B \cap \delta$ is uncountable and $\operatorname{dom}\left(\bar{p}_{\delta}^{0}\right) \cap \operatorname{dom}\left(\bar{p}^{2}\right)=$ $\operatorname{dom}\left(\bar{p}^{0}\right)$. Since $\left\langle p_{\beta, \delta}: \beta \in B \cap \delta\right\rangle$ forms a $\Delta$-system with root $p_{\delta}^{1}$ and since $\operatorname{dom}\left(\bar{p}_{\delta}^{0}\right)$ is countable, we have $\operatorname{dom}\left(p_{\beta, \delta} \backslash p_{\delta}^{1}\right) \cap \operatorname{dom}\left(\bar{p}_{\delta}^{0}\right) \neq \emptyset$ for only countably many $\beta \in B \cap \delta$. So pick a $\beta \in B \cap \delta$ such that $\operatorname{dom}\left(p_{\beta, \delta} \backslash p_{\delta}^{1}\right) \cap \operatorname{dom}\left(\bar{p}_{\delta}^{0}\right)=\emptyset$.

Define $r \in \mathbf{P}_{\kappa}$ as follows: 


$$
\begin{aligned}
& \operatorname{dom}(r)=\operatorname{dom}\left(r_{\beta}\right) \cup \operatorname{dom}\left(\bar{p}_{\delta}^{0}\right) \cup \operatorname{dom}\left(p_{\beta, \delta} \backslash p_{\delta}^{1}\right), \\
& r \mid \operatorname{dom}\left(r_{\beta} \cup \bar{p}_{\delta}^{0}\right)=r_{\beta} \cup \bar{p}_{\delta}^{0}, \\
& \text { and } \\
& r(\xi)=p_{\beta, \delta}(\xi) \text { for } \xi \in \operatorname{dom}\left(p_{\beta, \delta} \backslash \operatorname{dom}\left(r_{\beta} \cup \bar{p}_{\delta}^{0}\right)\right) .
\end{aligned}
$$

Then $r$ is a well-defined condition with the property that $r \leq r_{\beta}, \bar{p}_{\delta}^{0}$ and $p_{\beta, \delta}$. So $r$ forces that $\{\beta, \delta\} \in X / W$ and that $\{\beta, \delta\} \in K_{i}$, which is a contradiction.

If $\mathbf{P}_{\kappa}$ is as in the assumptions of Lemma 44, then $\mathbf{P}_{\kappa}$ is a $\mathbf{c}^{+}$-c.c. poset. If GCH holds in the ground model and $\lambda=\omega_{1}$, then our proof works if $\kappa \geq \aleph_{8}$. One can obtain the consistency of $\mathfrak{c} \rightarrow(\omega-\text { path })_{\omega_{1} /<\omega}^{2}$ with a smaller size of the continuum, but this is not essential for our purposes. Todorcevic has for example shown that, adjoining at least $\omega_{2}$ Cohen reals to a model of the Continuum Hypothesis, produces a model in which $\omega_{2} \rightarrow(\omega-\text { path })_{\omega /<3}^{2}$.

We have actually proved something apparently stronger than $\mathfrak{c} \rightarrow$ $(\omega-\text { path })_{\lambda /<\omega}^{2}$ in $V^{\mathbf{P}_{\kappa}}$, namely a relation denoted by $\mathfrak{c} \rightarrow(\omega-\text { path })_{\lambda /<3}^{2}$.

We do not know an answer to the following two problems concerning the $\omega$-path partition relation:

Problem 4 Is it for each integer $k>2$ consistent, for some infinite cardinal numbers $\kappa$ and $\lambda$, that $\kappa \nrightarrow \rightarrow(\omega \text {-path })_{\lambda /<k}^{2}$, but $\kappa \rightarrow(\omega \text {-path })_{\lambda /<k+1}^{2}$ ?

Problem 5 Is it consistent, for some infinite cardinal numbers $\kappa$ and $\lambda$, that for each $k<\omega, \kappa \nrightarrow \rightarrow(\omega-\text { path })_{\lambda /<k}^{2}$, but $\kappa \rightarrow(\omega-\text { path })_{\lambda /<\omega}^{2}$ ?

Theorem 45 (Todorcevic) If $Z F C$ is a consistent theory, then so is the theory $Z F C+\operatorname{cof}\left(\left\langle J_{\mathbb{R}}\right\rangle, \subset\right)=\aleph_{1}+\mathfrak{c} \rightarrow(\omega-\text { path })_{\omega_{1} /<\omega}^{2}$.

Proof Theorem 45 is an immediate consequence of Lemma 44: It is well known that if $\mathrm{CH}$ holds in the ground model, and $\mathbf{P}$ is e.g. Sacks or Prikry-Silver forcing, then (b) and (c) of the lemma hold for every $\kappa$. It is also known that adding any number of Sacks or PrikrySilver reals side-by-side with countable supports to a model of $\mathrm{CH}$, one obtains a model where the collection of meager sets whose Borel codes are from the ground model, is a cofinal subfamily of $J_{\mathbb{R}}$ (see $[\mathrm{M}])$. Since $\left|{ }^{\omega} \omega \cap V\right|=\aleph_{1}$, we get $\operatorname{cof}\left(\left\langle J_{\mathbb{R}}\right\rangle, \subset\right)=\aleph_{1}$ in the forcing extension.

\section{References}

[C] G. Choquet, Lectures in Analysis, Vol. 1, Benjamin, New York (1969). 
[Ci] J. Cichon, On two-cardinal properties of ideals, Transactions of the American Mathematical Society 314 (1989), 693 - 708.

[C-N] W.W. Comfort and S. Negrepontis, Chain conditions in topology, Cambridge University Press (1982).

[D] G. Debs, Stratégies gagnantes dans certains jeux topologiques, Fundamenta Mathematicae 126 (1985), 93-105.

[F-K] W.G. Fleissner and K. Kunen, Barely Baire Spaces, Fundamenta Mathematicae 101 (1978), 229-240.

[F] D.H. Fremlin, Cichon's diagram, in Séminaire d'Initiation á l'Analyse, Univ. Pierre et Marie Curie, Paris 23 (1985), 5.01-5.13.

[G-T] F. Galvin and R. Telgarsky, Stationary strategies in topological games, Topology and its Applications 22 (1986), 51-69.

[I] J.R. Isbell, The category of cofinal types. II, Transactions of the A.M.S. 116 (1965), 394-416.

[J] T. Jech, Multiple Forcing, Cambridge University Press (1986).

[J-M-P-S] W. Just, A.R.D. Mathias, K. Prikry and P. Simon, On the existence of large p-ideals, The Journal of Symbolic Logic (1990), 457-465.

[Ko] P. Koszmider, On Coherent Families of Finite-to-One Functions, The Journal of Symbolic Logic, to appear.

[K] K. Kunen, Random and Cohen reals, in Handbook of SetTheoretic Topology (Edited by K. Kunen and J.E. Vaughan), Elsevier Science Publishers (1984), pp. 887-911.

[L] R. Laver, Linear orders in ${ }^{\omega}(\omega)$ under eventual dominance, Logic Colloquium '78, North-Holland (1979), 299 - 302.

[L-M-S] J. Levinski, M. Magidor and S. Shelah, Chang's conjecture for $\aleph_{\omega}$, Israel Journal of Mathematics 69 (1990), 161-172.

[M] A. W. Miller, Some properties of measure and category, Trans. Amer. Math. Soc. 266 (1981), 93-114.

[S1] M. Scheepers Meager-nowhere dense games (I): n-tactics, The Rocky Mountain J. of Math. 22 (1992), to appear.

[S2] M. Scheepers A partition relation for partially ordered sets, Order 7 (1990), 41-64.

[S3] M. Scheepers, Meager-nowhere dense games (IV): n-tactics and coding strategies, preprint.

[T] R. Telgarsky, Topological games: on the 50-th anniversary of the Banach-Mazur game, Rocky Mountain Journal of Mathematics 17 (1987), 227-276.

[To1] S. Todorčević, Reals and Positive Partition Relations, in:Logic, Methodology and Philosophy of Science VII, edited by B. Marcus et al., Elsevier Science Publishers (1986), 159-169. 
[To2] S. Todorčević, Kurepa families and cofinal similarities, preprint, (December 1989).

[To3] S. Todorčević, Cofinal Kurepa Families, preprint, (November 1990).

[To4] S. Todorčević, Partitioning pairs of countable sets, Proceedings of the American Mathematical Society 111 (1991), 841-844.

[W] W.H. Woodin, Discontinuous homomorphisms of C(Omega) and Set Theory, Ph. D. dissertation, University of California, Berkeley (1984). 